\title{
Dynamics of multiply charged ions in intense laser fields
}

\author{
S. X. Hu ${ }^{*}$ and C. H. Keitel ${ }^{* *}$ \\ Theoretische Quantendynamik, Fakultät für Physik, \\ Albert-Ludwigs-Universität Freiburg, Hermann-Herder-Str.3, D-79104 \\ Freiburg, Germany
}

(October 23, 2018)

\begin{abstract}
We numerically investigate the dynamics of multiply charged hydrogenic ions in near-optical linearly polarized laser fields with intensities of order $10^{16}$ to $10^{17} \mathrm{~W} / \mathrm{cm}^{2}$. The weakly relativistic interaction is appropriately described by the Hamiltonian arising from the expansion of the Dirac equation up to the second order in the ratio of the electron velocity $v$ and the speed of light $c$. Depending on the charge state $Z$ of the ion the relation of strength between laser field and ionic core changes. We find around $Z=12$ typical multiphoton dynamics and for $Z=3$ tunneling behaviour, however with clear relativistic signatures. In first order in $v / c$ the magnetic field component of the laser field induces a Z-dependent drift in the laser propagation direction and a substantial Z-dependent angular momentum with repect to the ionic core. While spin oscillations occur already in first order in $v / c$ as described by the Pauli equation, spin induced forces via spin orbit coupling only appear in the parameter regime where $(v / c)^{2}$ corrections are significant. In this regime for
\end{abstract}


$Z=12$ ions we show strong splittings of resonant spectral lines due to spinorbit coupling and substantial corrections to the conventional Stark shift due to the relativistic mass shift while those to the Darwin term are shown to be small. For smaller charges or higher laser intensities, parts of the electronic wavepacket may tunnel through the potential barrier of the ionic core, and when recombining are shown to give rise to $\mathrm{keV}$ harmonics in the radiation spectrum. Some parts of the wavepacket do not recombine after ionisation and we find very energetic electrons in the weakly relativistic regime of above threshold ionization.

PACS Numbers: $32.80 \mathrm{Rm}, 42.50 \mathrm{~Hz}, 33.40+\mathrm{f}$ 


\section{INTRODUCTION}

Various techniques to generate ultrashort pulses such as chirped-pulse-amplification (CPA) [1] have been developed and perfected over the last years such that nowadays powerful laser pulses are available over a wide range of frequencies and pulse lengths up to intensities of $10^{21} \mathrm{~W} / \mathrm{cm}^{2}$ [2]. For intensities of $10^{16} \mathrm{~W} / \mathrm{cm}^{2}$ to $10^{19} \mathrm{~W} / \mathrm{cm}^{2}$ the electric field strength of the laser pulse is already comparable up to far stronger than the atomic unit field strength $\left(\sim 5.14 \times 10^{9} \mathrm{~V} / \mathrm{cm}\right)$ that is experienced by an electron on the first Bohr orbit of hydrogen and in fact those fields are already accessible in quite numerous laboratories worldwide in rather small table-top set-ups.

Especially for laser intensities till at about $10^{16} \mathrm{~W} / \mathrm{cm}^{2}$ there has been a lot of activity over the last two decades in intense nonrelativistic laser interactions with matter including atoms [3], molecules [4], clusters [5] and solids [6]. Many highly nonlinear optical phenomena such as high-order harmonic generation [7 10, above-threshold ionization (ATI) [11] and for higher frequencies and intensities stabilization [12], have attracted much attention for both experimentalists and theorists. Since optical laser fields of intensity $10^{21} \mathrm{~W} / \mathrm{cm}^{2}$ have become available in a rather short time, both theoretical and experimental activities moved quite quickly into the regime of fully relativistic dynamics up to around $10^{18} \mathrm{~W} / \mathrm{cm}^{2}$ to $10^{22} \mathrm{~W} / \mathrm{cm}^{2}$ 13 15 with experimental emphasis on free electron dynamics, QED effects and nuclear reaction processes [16,17]. There has been rather little activity however for neutral atoms interacting with optical laser fields of intensity $10^{16} \mathrm{~W} / \mathrm{cm}^{2}$ to $10^{17} \mathrm{~W} / \mathrm{cm}^{2}$ merely for the reason, that there is almost instantaneous ionization for those intensities and the free electron dynamics is quite well understood for a long time [18]. For higher frequencies ionization may be retained at those intensities however will also occur eventually for larger laser intensities due to the magnetic drift [13] with a likelyhood for magnetic recollisions being usually smaller than that in the laser polarisation direction [19.

For an ionic system one may have the unique possibility to apply relativistic near-optical 
laser field pulses and still allow for bound dynamics. The physical processes occuring will not merely scale with the ionic charge but be fundamentally different because of relativity and later QED governing the dynamics. Nowadays ions may be processed from essentially all existing atoms with abitrary charge state, absolute purity and quite high density by sending them through foils [20] while due to lasers high charge states have also been achieved as well however with limits in the width of the charge distribution and the absolute charge [21]. The mean electric field sensed by a ground state electron of hydrogenic uranium corresponds to $1.8 \times 10^{16} \mathrm{~V} / \mathrm{cm}$. Thus laser fields that are comparable in strength need have an intensity of order $10^{29} \mathrm{~W} / \mathrm{cm}^{2}$ and are thus far beyond reach nowadays. For those intensities the dynamics will be absolutely remote from that of hydrogen with say a laser field of $10^{14} \mathrm{~W} / \mathrm{cm}^{2}$ and we do not aim to make the corresponding comparison in this paper. We consider it however more interesting to carry out a comparision of the nonrelativistic laser atom interaction with the weakly relativistic dynamics of an ion of charge around 10 with laser fields of intensity $10^{16} \mathrm{~W} / \mathrm{cm}^{2}$ to $10^{17} \mathrm{~W} / \mathrm{cm}^{2}$ (see figure 1 ). Here relativity induces merely corrections and it is possible to identify the leading deviations to nonrelativistic dynamics as that of the laser magnetic field component, the break-down of the dipole approximation, spin-orbit coupling, zitterbewegung amd the relativistic mass shift. In earlier letters we have shown that the magnetic field component of the laser pulse induces an enhanced angular momentum and thus a reduced electron expectation value in the vicinity of the multiply charged ion [22] and the existence of spin signatures in bound electron dynamics and radiation [23].

In terms of applications multiply charged ions in relativistic laser fields appear also attractive for the generation of energic electrons and high harmonics. The kinetic energy of a laser-accelerated electron as characterised by the parameter $U_{p}$ does certainly increase with rising laser intensity. For harmonic generation especially it is necessary that the highly energetic electron interacts periodically with the nucleus, urging us to enhance the charge of the ionic core, i.e. the ionization potential $I_{p}$ correspondingly. As long as tunneling and recollisions can be assured with an appropriate relation of $U_{p}$ and $I_{p}$ the simultaneous increase of both parameters is obviously attractive as the cut-off energy of high harmonic 
generation is given by the sum of $I_{p}$ and the maximal kinetic energy at the time of recollision being of order $U_{p}$.

In this paper, we study the dynamics of multiply charged ions in intense lasers fields in the weakly relativistic parameter regime where terms up to $1 / c^{2}$ are still of significance and higher terms become negligible. For this purpose we solve numerically the two-dimensional time-dependent expansion of the Dirac equation which is exact up to all orders in $1 / c^{2}$ and which was first derived by Foldy and Wouthuysen [24]. Different weakly relativistic effects arise and we can investigate each by solving the dynamics and comparing the situations in which we include or neglect the corresponding part of the Hamiltoninan associated with the effect of interest. As a function of the ionic charge relative to the laser field intensity the electric and magnetic field components of the laser field will be shown to be either strong enough to lead to ionization in the polarization and propagation directions or give rise to interesting structures in the near vicinity of the ionic core. We point out the role of the spin in inducing stronger binding of the electronic wavepacket and multiphoton spectral line splitting due to spin orbit coupling. This is compared with the situation via the Pauli equation where spin induced forces are neglected. We further show that the relativistic mass shift induces a significant shift of especially the highly excited eigenenergies of the ion with respect to the conventional Stark shift and point out the consequences for the spectral lines. For relatively high intensities special emphasis is placed on the tunneling regime in the weakly relativistic regime. For multiply charged ions we find that both tunneling and recollisions are still possible and indicate harmonic generation in the keV regime. We note however that the plateau of the harmonic spectrum, as well known in the nonrelativistic regime, is tilted increasingly in the relativistic regime with rising charge. This deviation to conventional high harmonic spectra is not too surprising as an electric wavepacket attempting to recollide due to a phase shift of the electric field in the laser polarization direction may still partly or totally miss the ionic core due the magnetic drift in the laser propagation direction, especially for long recollision times. In the above threshold spectra we find high electron energies in the $\mathrm{keV}$ regime which are separated by the photon energy of the applied laser field. 
This paper is arranged as follows: In section II, we derive the Hamiltonian of interest along the lines of Foldy and Wouthuysen, then describe the numerical methods for solving the corresponding dynamical equation and present the details for computing the observable quantities of interest. In subsection $\operatorname{III}(\mathrm{A})$ we then present effects up to first order in $1 / c$ as those induced by the magnetic field component of the laser field. In subsection III(B) we investigate the relativistic corrections to the Stark shift for laser-driven ions followed by a study of spin induced forces and the consequent splitting of spectral features in III(C). Subsequently, relativistic high order harmonic generation is discussed in III(D), as well as the photoelectron spectra in subsection III(E). Finally, a conclusion is drawn.

\section{PRELIMINARY CONSIDERATIONS}

In this more technical section we present subsequently the Hamiltonian describing our system of interest, explain how we solve the corresponding dynamical equation numerically via the split-step mechanism and finally quantify the observables to be investigated in the following section.

\section{A. System and Hamiltonian}

We are interested throughout this article in the dynamics of multiply charged ions of charge state up to $Z=12$ with moderate laser intensities $\left(10^{16} \sim 10^{17} \mathrm{~W} / \mathrm{cm}^{2}\right)$ and near optical frequencies for the $\mathrm{KrF}$ (248nm; 0.1838 a.u.) laser system and the doubled Nd:glass laser frequency $(527 \mathrm{~nm} ; 0.0866 \mathrm{a.u}$.). The maximum velocity of electron wavepackets reaches the order $v=0.1 c$, where $c=137.036$ denotes the speed of light in atomic units. We are therefore entitled to consider the Dirac equation up to second order in $v / c$ and have confirmed that for our parameters high order corrections do not play a role. One may derive this Hamiltonian via various unitary transformations along the lines of the Foldy-Wouthuysen (FW) expansion [24] of the Dirac equation or alternatively, with the same result, find the first order relativistic corrections to the Pauli equation [25]. As opposed to nonrelativistic 
treatments we need to include at least two dimensions in the calculation as the magnetic field component of the laser pulse may induce a significant drift in the laser propagation direction. There is a spin-induced force in the magnetic field direction [26], i.e. in the remaining third dimension, but the influence is small for the observables and the parameters of interest here.

Our working Hamiltonian $\mathbf{H}_{\mathbf{F w}}$ involves a series of relativistic corrections to the usual nonrelativistic Schrödinger Hamiltonian. One well-known correction term in $v / c$ to the Schrödinger equation is the additional term in the Pauli equation representing the coupling of the laser magnetic field to the spin degree of freedom of the electron wavepacket. The second order terms include the spin orbit coupling, i.e. the feed-back of the oscillating spin to the electron motion, as well as the leading relativistic mass shift term and Zitterbewegung. Terms of order $O\left(1 / c^{3}\right)$ do not play a role for the parameters employed though would certainly be necessary to include for the fully relativistic regime involving more intense laser fields and higher charged ions. The main advantage of using this equation in comparison to the full Dirac equation is the possible isolation of the influence of each physical mechanism arising. In addition this equation does not limit us numerically to use high frequency lasers as necessary so far with the full Dirac equation [15].

For the circumstances with laser parameters described above, the FW Hamiltonian $(2 \times 2$ matrix) of a bound electron in a strong laser field can be written (in atomic units [27]) as

$$
\begin{aligned}
& \mathbf{H}_{\mathbf{F W}}=\mathbf{H}_{\mathbf{0}}+\mathbf{H}_{\mathbf{p}}+\mathbf{H}_{\mathbf{k i n}}+\mathbf{H}_{\mathbf{D}}+\mathbf{H}_{\mathbf{s o}} \\
& \mathbf{H}_{\mathbf{0}}=(\mathbf{p}+\mathbf{A}(z, t) / c)^{2} / 2+V(x, z) \\
& \mathbf{H}_{\mathbf{P}}=\sigma \cdot \mathbf{B}(z, t) / 2 c \\
& \mathbf{H}_{\mathbf{k i n}}=-\mathbf{p}^{4} / 8 c^{2} \\
& \mathbf{H}_{\mathbf{D}}=\operatorname{div} \mathbf{E}^{\prime}(x, z, t) / 8 c^{2} \\
& \mathbf{H}_{\mathbf{s o}}=i \sigma \cdot \operatorname{curl} \mathbf{E}^{\prime} / 8 c^{2}+\sigma \cdot \mathbf{E}^{\prime} \times \mathbf{p} / 4 c^{2} .
\end{aligned}
$$

Here $\mathbf{H}_{\mathbf{0}}$ denotes the standard nonrelativistic Hamiltonian in Schrödinger form, where $\mathbf{p}=\left(p_{x}, 0, p_{z}\right)=(-i \partial / \partial x, 0,-i \partial / \partial z)$ is the two-dimensional momentum operator and $\mathbf{A}(z, t)$ is the time-spatial dependent vector potential of the laser field $\mathbf{E}(z, t)$, which is 
linearly polarized along the $x$-axis and propagates in $z$-direction. For the vector potential we include the magnetic field component and do not apply the dipole approximation, urging us to perform a two-dimensional numerical integration in the $x-z$ plane. Since $\mathbf{H}_{\mathbf{0}}$ all other Hamiltonians are $2 \times 2$ matrices, they need be considered as multiplied by the unity matrix I even if not shown explicitely throughout this paper. We consider multiply charged ions in the single active electron approximation [28] which are preionized by several or more than ten electrons and thus are easily availabe today via lasers [21] or with highest accuracy via shooting the atoms through thin foiles [20]. Those are well described by the soft-core potential 29] to model the Coulomb field experienced by the active electron of a multiply charged ion, i.e.

$$
V(x, z)=-k / \sqrt{q_{e}+x^{2}+z^{2}} .
$$

The parameter $k$ is a function of the effective number of positive charges $Z$ as sensed by the electron. $q_{e}$ compensates for the effect of possible inner electrons and reduced distances of the electronic wavepacket to the ionic core in two- rather three-dimensional calculations. $k$ may be adapted such that we obtain the correct ionization energy for the system of interest with effective charge of the ionic core $Z$ and charge of the ion $Z-1$. The static field of the ionic core is expressed by the gradient of the potential $-\nabla V(x, z)$ and $\mathbf{E}^{\prime}(x, z, t)$ stands for the sum of this field plus the laser field $\mathbf{E}(z, t)$. The following term $\mathbf{H}_{\mathbf{P}}$ in Eq.(1) indicates the coupling of the laser magnetic field $\mathbf{B}$ to the electronic spin as described by the Pauli matrix $\sigma$. The sum $\mathbf{H}_{\mathbf{0}}+\mathbf{H}_{\mathbf{P}}$ leads to the Hamiltonian in the well known Pauli equation. Further in Eq. (1) $\mathbf{H}_{\text {kin }}$ denotes the leading term for the relativistic mass increase, and $\mathbf{H}_{\mathbf{D}}$ is the well-known Darwin term. Finally the last term in the Hamiltonian $\mathbf{H}_{\text {so }}$ stands for the spin-orbit coupling. Considering our central potential $V(x, z)$ the first term of $\mathbf{H}_{\text {so }}$ in Eq. (1) disappears because $\nabla \times(-\nabla V(x, z))=0$ and the contribution due to the laser field is of order $1 / c^{3}$. Thus, the spin-orbit coupling term becomes

$$
\mathbf{H}_{\text {so }}=\sigma \cdot \mathbf{E}^{\prime} \times \mathbf{p} / 4 c^{2}=\sigma \cdot \mathbf{E} \times \mathbf{p} / 4 c^{2}+f(x, z) \sigma \cdot \mathbf{L}
$$


with $f(x, z)=-k\left(q_{e}+x^{2}+z^{2}\right)^{-3 / 2} / 4 c^{2}$ and where $\mathbf{L}=\mathbf{r} \times \mathbf{p}=\left(0, z p_{x}-x p_{z}, 0\right)$ is the orbital angular momentum, of which only the component along the y-direction is nonzero. The origin of spin-orbit coupling can alternatively be viewed also as being due to the interaction between the magnetic moment of the electron and the magnetic field $\mathbf{B}^{\prime}$ due to the motion of the positively charged core as sensed by the electron in its own rest frame.

\section{B. Dynamics and Numerical Approach}

We investigate the dynamics of multiply charged ions exposed to an intense laser field through solving the following dynamical equation, involving the previously derived Hamiltonian $\mathbf{H}_{\mathbf{F w}}$ in Eq. (1) (for the convenience, we use the usual atomic units throughout this paper [27]):

$$
i \frac{\partial}{\partial t}\left(\begin{array}{c}
\Psi_{u p}(x, z, t) \\
\Psi_{\text {down }}(x, z, t)
\end{array}\right)=\mathbf{H}_{\mathbf{F W}}\left(\begin{array}{c}
\Psi_{u p}(x, z, t) \\
\Psi_{\text {down }}(x, z, t)
\end{array}\right)
$$

The wave function has two components corresponding to spin-up and spin-down polarization of the electron and $\mathbf{H}_{\mathbf{F W}}$ is consequently a $2 \times 2$ matrix operator. The coupling to negative energy states as included in conventional Dirac theory is negligible in second order in $v / c$. The laser field is assumed to be linearly polarized along the $x$-axis so that the vector potential $\mathbf{A}(z, t)$ of the laser field may be of the form

$$
\mathbf{A}(z, t)=\left(A_{x}(z, t), 0,0\right)
$$

where the $z$-dependence of the vector potential reflects the propagation of the laser pulse in the $z$ direction. The spatial dependence of the vector potential indicates that the magnetic component of the laser field $B=\nabla \times \mathbf{A}(z, t) / c \neq 0$ is included and we do not (and can not) carry out the dipole approximation. We choose the vector potential $A_{x}(z, t)$ to be

$$
A_{x}(z, t)= \begin{cases}-\frac{c E_{0}}{\omega t_{o n}}\left[(t-z / c) \sin (\omega t-\omega z / c)+\frac{1}{\omega} \cos (\omega t-\omega z / c)\right] & 0<t-z / c \leq t_{\text {on }} \\ -\frac{c E_{0}}{\omega} \sin (\omega t-\omega z / c) & t_{\text {on }}<t-z / c<t_{p}\end{cases}
$$


which is associated with a linearly polarized laser field with electric field $E_{x}$ and magnetic field $B_{y}$ components

$$
E_{x}(z, t)=B_{y}(z, t)= \begin{cases}E_{0} \frac{t-z / c}{t_{o n}} \cos (\omega t-\omega z / c) & 0<t-z / c \leq t_{o n} \\ E_{0} \cos (\omega t-\omega z / c) & t_{o n}<t-z / c<t_{p}\end{cases}
$$

being oriented in the $x$ and $y$ direction, respectively and propagating both in phase in the $z$ direction. Here, $E_{0}$ and $\omega$ are the maximal amplitudes of both fields and the angular frequency of the laser field, respectively. Further $t_{\text {on }}$ is associated with the linear rising time of the laser pulse, i.e. 0 is the beginning of the turn-on and $t_{\text {on }}$ the end of it. We note that the vector potential Eq. (6) may not be continuous at the end of the turn-on phase, however it is when $t_{\text {on }}$ is chosen such that $\omega\left(t_{o n}-z / c\right)=(m+0.25) 2 \pi$ with $\mathrm{m}$ being an arbitrary integer. The measurable electric field strength however is always continuous. After the turn-on phase the pulse is assumed to have a constant amplitude till time $t_{p}$. Obviously a realistic pulse will turn off afterwards smoothly, however for all observables of interest here this phase is of no interest and numerical calculations usually terminate at $t_{p}$.

Since the laser field is linearly polarized, the interaction term involves a term of the form $\mathbf{p} \cdot \mathbf{A}(\mathbf{z}, \mathbf{t}) / \mathrm{c}=\mathrm{p}_{\mathrm{x}} \mathrm{A}_{\mathrm{x}}(\mathrm{z}, \mathrm{t}) / \mathrm{c}$. which means no coupling between momentum and coordinate space. This is because the term couples only the $x$ component of the momentum with a function which is dependent on $z$ but not on $x$. Thus, we can still apply the usual splitoperator algorithm [30] to solve the two-dimensional time-dependent matrix equation (4) via

$$
\begin{aligned}
\left(\begin{array}{c}
\Psi_{\text {up }}(x, z, t+\Delta t) \\
\Psi_{\text {down }}(x, z, t+\Delta t)
\end{array}\right)= & \exp \left[-i \Delta t\left(p^{2} / 4-p^{4} /\left(16 c^{2}\right)\right) \mathbf{I}\right] \\
& \times \exp \left[-i \Delta t\left(\left(\mathbf{p} \cdot \mathbf{A} / c+A^{2} / c^{2}\right) \mathbf{I}+\mathbf{H}_{\mathbf{D}}\right)\right] \times \exp \left[-i \Delta t\left(\mathbf{H}_{\mathbf{P}}+\mathbf{H}_{\text {so }}\right]\right. \\
& \times \exp \left[-i \Delta t\left(p^{2} / 4-p^{4} /\left(16 c^{2}\right)\right) \mathbf{I}\right] \times\left(\begin{array}{c}
\Psi_{\text {up }}(x, z, t) \\
\Psi_{\text {down }}(x, z, t)
\end{array}\right) .
\end{aligned}
$$

Here, $\Delta t$ denotes the time step and the unit matrix operator is described with $\mathbf{I}$. All expo- 
nential operators except the term $\exp \left[-i \Delta t\left(\mathbf{H}_{\mathbf{P}}+\mathbf{H}_{\mathbf{s o}}\right]\right.$ are diagonal and we apply the split evolution operator on the wave function with the help of Fourier transforming between the coordinate representation and the momentum representation. Consequently all derivative operator can be transformed into multiplications with constants. For non-diagonal exponential operators, we usually need to diagonize them. Fortunately, the non-diagonal operator $\exp \left[-i \Delta t\left(H_{P}+H_{s o}\right]\right.$ involved here only depends on the specific Pauli matrix $\sigma$, so that we can carry out the Taylor expansion for this exponential operator up to the order of interest here. Regarding the interaction term $p_{x} A_{x} / c$ special care is needed as mentionned above. Here we do the Fourier transformation only for the x-coordinate because of the z-coordinate dependence of the vector potential $A_{x}(z, t)$. This however is sufficient and the exponential function of operators of interaction terms on the wave function ends up being merely a sequence of Fourier transformations and c-number multiplications. Because of the splitting of the total Hamiltonian in the exponent we introduce an error following the Baker-Hausdorff formula because the split terms do generally not commute. The error of this algorithm is of order $(\Delta t)^{3}$ between every successive time step. Thus, a small time step can ensure to get accurate results; and we have not experienced any problem with numerical convergence in the regime of interest here.

From the numerical point of view we first solve for the eigenstates of the bound electron in the ionic core potential by using the so-called "spectral" method [31. In fact, we choose a testing wave function without any symmetries to propagate on the two-dimensional potential. This way we obtain all symmetric and asymmetric eigenstates of the system. As an example, figure 2 shows the energy-level structure of a model hydrogen-like ion $M g^{11+}$ of which the single active electron experiences the nucleus with charges $\mathrm{Z}=12$. Choosing $\mathrm{k}=80.32$ and $q_{e}=1.0$ we get the corresponding ground-state energy $-72 \mathrm{au}$. Note that it is not our purpose to present an exact quantitative model of the ionic level structure of an ionic system. We model single electron ions with a softcore rather than a Coulomb potential and can adapt the ground state energy by choosing $k$ and $q_{e}$. With the assumption of a smooting around the ionic core, we deviate from the exact Coulombic potential and the system may 
also be considered as an active electron plus an ionic core where lower shells are filled and inactive. In the intensity range where level structures are important we thus can only make qualitative statements for the dynamics if we want to associate it with a particular realistic ion. In the tunneling regime however where structure becomes less important and only the correct ground state is significant we can be quantitative as well.

The ground-state wave packet of our model ion relates to a symmetric s-state wavepacket, and also the second excited-state wave packet (see figure $2 \mathrm{~b}$ ). The first, third and fourth excited-states are asymmetric (especially not s-states) in space. This system will be applied to evaluate the relativistic Stark shift in section III(B). After obtaining the eigenstates $\Phi_{n}(x, z)$, we may use the above split-step operator in Eq.(8) to investigate the evolution of multiply charged ions under the irradiation of external intense lasers. When the laser pulse is turned on, the system is assumed to evolve from the spin-polarized ground state, that is, the initial condition $\Psi_{u p}(x, z, t=0)=\Phi_{1}(x, z)$ and $\Psi_{\text {down }}(x, z, t=0)=0$.

\section{Observables of interest}

With the knowledge of the time-dependent wavefunction we are in the position to cal-

culate the spatial probability distribution $|\Psi(x, z, t)|^{2}=\left|\Psi_{u p}(x, z, t)\right|^{2}+\left|\Psi_{\text {down }}(x, z, t)\right|^{2}$ and the expectation value of any observable $\mathcal{O}$ associated with the system via

$$
\langle\mathcal{O}(t)\rangle=\int_{x_{\min }}^{x_{\max }} \mathrm{d} x \int_{z_{\min }}^{z_{\max }} \mathrm{d} z\left(\Psi_{u p}(x, z, t), \Psi_{\text {down }}(x, z, t)\right) \mathcal{O}(x, z, t)\left(\begin{array}{c}
\Psi_{u p}(x, z, t) \\
\Psi_{\text {down }}(x, z, t)
\end{array}\right)
$$

Here the integral turns into a sum in our case as the wavefunction is given on an equidistant grid from $x_{\min }$ to $x_{\max }$ in the polarization direction and from $z_{\min }$ to $z_{\max }$ in the propagation direction.

Particular interest in this article is placed on the spatial average values $\langle x(t) \mathbf{I}\rangle$ and $\langle z(t) \mathbf{I}\rangle$ of the wavepacket and because of their relevance for the radiation spectrum on the 
accelerations in the polarization direction $a_{x}(t)=\langle\ddot{x}(t) \mathbf{I}\rangle$ and in the propagation direction $a_{z}(t)=\langle\ddot{z}(t) \mathbf{I}\rangle$ via Eq. (9).

The radiation spectrum is generally given by a rather complex function of the accelerations and velocities in all spatial directions (see e.g. Eq. 10.7 in the first review in [3]). For simplicity we here restrict ourselves to an observation direction perpendicular to the $2 \mathrm{~d}$ plane of motion, i.e. the $y$ direction. Also we are mostly interested in the dominating part of the radiation spectrum in the weakly relativistic regime which is polarized in the polarization direction of the laser field. In the far field spectrum this is proportional to the squared Fourier transform of simply the acceleration $a_{x}(t)=\langle\ddot{x}(t) \mathbf{I}\rangle$. Also for the purpose of studying weakly relativistic signatures of the spectra it is interessing to study the less intense spectrum which is polarised in the laser propagation direction and governed by $a_{z}(t)=\langle\ddot{z}(t) \mathbf{I}\rangle$.

Under the assumption of including relativistic corrections up to second order in $v / c$ it is not sufficient for $a_{x}(t)$ to consider the gradient of the potential only as in the nonrelativistic regime. We rather find in the weakly relativistic limit

$$
\begin{aligned}
\ddot{x} & =\sqrt{1-\dot{x}^{2} / c^{2}-\dot{z}^{2} / c^{2}} \times\left(-\frac{\partial V(x, z)}{\partial x}+\frac{\dot{x}}{c}\left(\frac{\dot{x}}{c} \frac{\partial V(x, z)}{\partial x}+\frac{\dot{z}}{c} \frac{\partial V(x, z)}{\partial z}\right)\right) \\
& =\left(1+\frac{3}{2 c^{2}} \frac{\partial^{2}}{\partial x^{2}}+\frac{3}{2 c^{2}} \frac{\partial^{2}}{\partial z^{2}}+O\left(1 / c^{4}\right)\right) \times\left(-\frac{\partial V(x, z)}{\partial x}\right) \\
\ddot{z} & =\left(1+\frac{3}{2 c^{2}} \frac{\partial^{2}}{\partial x^{2}}+\frac{3}{2 c^{2}} \frac{\partial^{2}}{\partial z^{2}}+O\left(1 / c^{4}\right)\right) \times\left(-\frac{\partial V(x, z)}{\partial z}\right)
\end{aligned}
$$

where for the transformation to the second part of the equation we substituted the time derivatives via $\mathrm{d} x / d t=d H_{F W} / d p_{x}$ and $\mathrm{d} z / d t=d H_{F W} / d p_{z}$ and will neglect the higher order terms in $O\left(1 / c^{4}\right)$. We note that the first term in the right-hand side is just the nonrelativistic limit, and the following two terms correspond to weakly relativistic corrections which will be retained for the calculation of spectra. For the evaluation of the spectrum of interest the operators in Eq. (10) need just be inserted in Eq. (9) and then to be Fourier transformed.

We now turn to the technical aspects for the evaluation of the photoelectron spectra. Since the expected ionization is equivalent to a proportion of the electronic wave function 
leaving the vicinity of the ionic core and propagating outwards towards the (unphysical) boundaries of the numerical grid, we must avoid reflections of the wave function at those boundaries. This is achieved by absorbing all parts of the wave function approaching the boundaries by a $\cos ^{\frac{1}{8}}$ mask function [32], which results in a decrease of the norm of the wave function within the spatial box. While quite often only the part in the vicinity of the ionic core interests, it is here the opposite as we care only for the part of the wavefunction approaching the absorbing boundaries. Assume that we have calculated the wavepacket dynamics up to time $t_{f}$ in our finite box. Then we will have information about the photoelectron spectrum from what has been absorped till this time at the boundaries at all intermediate times $t_{\alpha}$ and from the ionized part of the wavefunction still in the box. We begin by treating the absorbed electron flux, say $\Psi_{\text {flux }}\left(x, z, t_{\alpha}\right)$ at time $t_{\alpha}$ after entering the area of the boundaries. As this part will certainly not be influenced by the ionic core we will assume this to propagate to the end of interaction as a free particle (for details see [33]).

At the end of the interaction, at $t_{f}$, we also have to include the ionized part of the final spatial wave function still in the box. In order to obtain this from the full wave function it will be modified as follows, for example for the spin-up part of the wave function

$$
\Psi_{\text {out }}\left(x, z, t_{f}\right)=\left\{\begin{array}{lc}
0 & |x|<X_{I} \\
\sin ^{2}\left(\pi\left(|x|-X_{I}\right) / 2 X_{0}\right) \Psi_{u p}\left(x, z, t_{f}\right) & X_{I} \leq|x| \leq X_{I}+X_{0} \\
\Psi_{u p}\left(x, z, t_{f}\right) & |x|>X_{I}+X_{0}
\end{array}\right.
$$

Here $X_{I}$ represents a range which may be related to the ionic radius within which the electron may be considered bound and we will ignore this part of the wavepacket as we are only interested in the ionized part of the wave packet. We call $X_{0}$ the sliding range for the final wavefunction, where with increasing distance from the ionic core the wavefunction will contribute more to the photoelectron spectrum. Everything beyond $X_{I}+X_{0}$ can be considered as fully ionized and will fully contribute. The momentum wave function of the ionized electron can then be obtained by fast Fourier transforming both the spatial wave function $\Psi_{\text {out }}\left(x, z, t_{f}\right)$ and the freely propagated $\Psi_{\text {flux }}\left(x, z, t_{\alpha}\right)$ with respect to $\mathrm{x}$. We note that the main part of the electron distribution is ejected along the polarization direction 
in our range of parameters, so that we focus here on the photoelectron spectrum in this direction even though it is analogous in the z-direction. The momentum wave function describing the emitted electron distribution in $\mathrm{x}$ direction can be thus expressed as

$$
\begin{aligned}
\Psi_{p}\left(p_{x}, z, t_{f}\right)= & F_{F} T_{x}\left[\Psi_{\text {out }}\left(x, z, t_{f}\right)\right] \\
& +\sum_{t_{\alpha}} e^{-i p_{x}^{2}\left(t_{f}-t_{\alpha}\right) / 2} F_{F}\left[\Psi_{\text {flux }}\left(x, z, t_{\alpha}\right)\right] .
\end{aligned}
$$

Here the symbol ' $F F T_{x}$ ' means fast Fourier transform of the wave function with respect to the $\mathrm{x}$-coordinate, and $p_{x}$ denotes the electron momentum along the $\mathrm{x}$-axis. The summation in the above equation is for all times $t_{\alpha}$ during which the absorped part of the wave packet is detected. The kinetic energy spectrum of the photoelectron can then be obtained by integrating the momentum wave function over the z-coordinate, i.e. $P\left(\epsilon_{x}, t_{f}\right) \simeq\left(1+\epsilon_{x} / c^{2}\right) / \sqrt{2 \epsilon_{x}}\left|\int \Psi_{p}\left(p_{x}, z, t_{f}\right) d z\right|^{2}$. The prefactor has been derived via $\mathrm{d} p_{x} / \mathrm{d} e_{x}$ where the kinetic energy of the photoelectrons has been approximated via $\epsilon_{x} \simeq p_{x}^{2} / 2-p_{x}^{4} / 8 c^{2}$ in atomic units, including only weakly relativistic corrections in second order. The same procedure is carried out for the spin-down wave function and to obtain the total photoelectron spectrum, we sum over both polarisations.

\section{RESULTS AND DISCUSSIONS}

In this section we present and discuss the role of the magnetic laser component (A) and of the relativistic mass effects (B) on dynamics and radiation of laser driven ions. In (C) spin effects are investigated regarding the Pauli $\sigma \mathbf{B}$ term that merely induces spin oscillations and the second order $\sigma \mathbf{L}$ term which is responsible for spin induced forces. In (D) harmonic generation is studied in the weakly relativistic regime and in (E) highly energetic above threshold ionization.

\section{A. Magnetic field effects}

For neutral atoms interacting with moderately intense laser pulses, the magnetic component of the laser field can usually be ignored in the evaluation of the wavepacket dynamics. 
Once however the velocity of the electron wavepacket becomes nonnegligible as compared to the velocity of light the Lorentz force $(\mathbf{v} / c) \times \mathbf{B}$ may not be ignored as compared to the electric field force of the laser field. This generally is the case when the relativity parameter $r=e E /(m \omega)$ is not neglible as compared to $c$, i.e. at least $1 \%$ depending on the observable of interest.

The magnetic field component of a linearly polarized laser field induces a drift in the laser propagation direction [18] which may be strong enough to induce instantaneous ionization even for high laser frequencies [13]. There is a regime however where the laser field and correspondingly the drift is weak enough such that the attraction of singly charged ions can still compensate for it and induce recollisions along the propagation direction [19]. If one does not want to be restricted in the laser field intensity, a more elegant solution to overcome the drift and avoid strong ionization would be to employ multiply charged ions.

In the following we focus on the magnetic field (B-field) induced time evolution of the electronic wavepacket in the vicinity of and away from the ionic core, and since second order relativistic effects turn out to be small for the employed laser and ion parameters, the Hamiltonian $H_{F W}$ need only include the term $H_{0}$. Thus, we numerically solve the two-dimensional time-dependent Schrödinger equation but beyond the usual dipole approximation and including the laser magnetic field, and the vector potential $A=A(z, t)$ consequently depends on both $\mathrm{t}$ and $\mathrm{z}$.

In fig. 3 we have displayed the dynamics of single electron ions with charge $Z=3$ in a) and $Z=4 \mathrm{in} \mathrm{b}$ ) in a laser field with a wavelength of $248 \mathrm{~nm}$ and an intensity $1.2 \times 10^{17} \mathrm{~W} / \mathrm{cm}^{2}$ during the pulse duration of 10-cycle constant amplitude. We have merely displayed the dynamics of the center of mass of the wavepacket $(<x(t)>,<z(t)\rangle)$ in order to compare with our classical intuition. For $Z=1$ and $Z=2$ we would have almost complete ionization for the laser intensity applied and the figure would ressemble that of a classical free electron drifting with its usual "zick-zack" in the laser propagation direction [18]. For $Z=3$ we see in Fig. 3a) that the ionic core starts to seriously compete with the drift imposed by the laser field, i.e. there is substantial ionization in the laser polarization and propagation direction 
however also a significant part can still be kept close to the ionic core. Interesting to see is that parts of the wavepacket may move opposite to the laser propagation direction similar to the magnetic recollisions advocated earlier [19]. We have also considered the center of mass motion restricted to a smaller area around the vicinity of the nucleus and found dynamics ressembling the "figure of 8 motion" known in the frame where the drift is transformed away. Simulations of the full wave packet have confirmed the dynamics towards and away from the nucleus in the laser propagation direction and the significant amount of ionization.

A different situation occurs in Fig 3b) for $Z=4$ when the ion charge is high enough to avoid substantial ionization and to allow for weakly relativistic bound dynamics. The electronic wave packet will oscillate around the ionic core with a particularly high velocity in its close vicinity. At the times when the electronic wave packet approaches the ionic core, the magnetic field component also turns out to be large. Therefore an extremely strong Lorentz push of the wave packet around the ionic core in the laser propagation direction arises and results in a low electron expectation value close to the ionic core [22]. The "open diamonds" represent the case without magnetic field, i.e. the situation within the dipole approximation. Here the electronic wave packet does merely carry out one-dimensional oscillations along the laser polarization direction, i.e. along the x-axis, as expected. However, when the magnetic component of the laser field is included in the calculation, the electronic wave packet is pushed towards the laser propagation direction (z-axis) as visible from the full circles. This magnetically induced pressure on the electronic wave packet is significantly increased around the ionic core as seen in the figure 3b). It results in a low electron probability in the vicinity of the ionic core. This can be interpreted as a hole around the nucleus at $(\mathrm{x}=0, \mathrm{z}=0)$ as indicated by the arrow in figure $3 \mathrm{~b}$ ). We need emphasize that we do not put forward holes from knocking out complete inner shell electrons from multi-electron systems. We rather discuss the strong reduction of the wave packet expectation value of single active electrons near the nucleus. For a multi-electron system, however, inner-shell electrons are likely to be more affected by the magnetically and ionic core induced Lorentz push investigated here, simply because the attraction from the nucleus is even larger then. 
It may be obvious that the electron velocity near the nucleus is high for multiply charged ions. However, we need explain why the magnetic field, also necessary for the Lorentz force, is significant at the time of the return of the electronic wave packet to the nucleus. When the electronic wave packet returns to the nucleus with essentially maximum velocity, the amplitude of the magnetic component of the laser field should become nearly zero. This is because velocity and force are generally phase shifted by $\pi / 2$. However, due to the existence of the multiply charged ionic core and the increasing field intensity during the turn-on of the laser field, an important phase lag can be developed during the laser pulse turn-on, which is very critical for the hole formation around the ionic core. During the laser pulse turn-on the electronic wave packet does not move like a quasi-free particle because of the tight binding of the ionic core. When the electronic wave packet is dragged outwards by the laser force, the strong ionic core tries to attract it, which results in an important phase lag between the wave packet motion and the laser field. Usually, when the wave packet returns to the nucleus, this effect is again compensated because this time the ionic core accelerates the electric wave packet towards it. However, because of the increasing field in the turn-on phase of the laser pulse, the laser force is stronger then, the Coulomb force comparably weaker and the phase lag consequently only partially compensated. Once the pulse is at full intensity, the phase lag remains almost unchanged till the field maximal amplitude begins to decrease again.

In the following we investigate the electronic relaxation dynamics after the laser pulse arising from the reduced circular motion around the ionic core. For this purpose, we allow the "hollow" ion to evolve freely for a time period of thirty laser cycles after the laser-ion interaction and during this time observe its relaxation dynamics and radiation. We note that the hollowing out of the ion due to the magnetic field will be reversed step by step in space during the relaxation process. In figure 4.a we have shown the relaxation radiation accompanying this process for the same potential and laser field parameters as in figure 3b). Apparently, the reverse process to magnetically induced hollowing out of the ion gives rise to $\mathrm{x}$-ray emission with distinct peaks up to $\sim 230 \mathrm{eV}$ photon energy. The main peak 
corresponds to the transition between the Stark-shifted first excited state $\mid 1 e>$ and the ground state $\mid g>$, as indicated in figure 4.b. Further the $122.67 \mathrm{eV}$ peak is related to the transition $|4 e>\rightarrow| g>$. Finally we note, that the hollowing out of the ion may be optimized, by adjusting the interaction parameters such that the Lorentz force $\mathbf{v} / c \times \mathbf{B}$ becomes maximal near the nucleus.

\section{B. Relativistic Stark shift}

In this subsection, we investigate the role of relativistic corrections to the Stark shift as visible in the positions of the energy levels of intense laser driven ions. For this situation we choose the Hamiltonian $H_{F W}=H_{0}+H_{P}+H_{k i n}$. We find that the Pauli term has no notable effect on the shift of energy levels but we include it for the sake of inclusion of all first order relativistic effects. The leading second order term in the weakly relativistic regime is generally $H_{k i n}$ which may be associated with a relativistic mass-increase. We note that the Darwin term is insignificant in its effect compared to the mass increase and in fact also to spin-orbit coupling. Since in this subsection we are not interested in line splitting but in the total shift of spectral components, we do not include $H_{\text {so }}$ here. The effect of the mass increase in intense laser driven atoms has been discussed before and associated with an enhancement of stabilization [34] and with Doppler shifts of harmonics [35]. Here we focus however on the multiphoton regime and how the ionic bound energy levels are shifted in addition to the conventional Stark shift.

We choose the model $\mathrm{Z}=12$ ion as described in section $\mathrm{II}(\mathrm{B})$ as target. The laser parameters involved are the intensity $7 \times 10^{16} \mathrm{~W} / \mathrm{cm}^{2}$, the wavelength $527 \mathrm{~nm}$, and a pulse including a 5.25-cycle turn-on phase and 100-cycle with constant amplitude. The whole radiation spectrum is displayed in figure 5 , in a) without including $H_{k i n}$ in the full Hamiltonian and in (b) with including it in the solution of the dynamical equation. The relativistic signatures are small on this scale, however, it is interesting to note that X-rays via hundreds of photons of the applied laser frequency may be generated due to resonances in the multiphoton 
regime.

We note further numerous resonant structures due to multiphoton transitions among the ionic bound levels in addition to smaller peaks displaced by up to few photon energies of the applied laser field to the resonant lines. In order to have a clearer picture of the structure and the deviation due to the relativistic mass shift, we have depicted the enlargements of the first three dominant resonant spectral structures in figures 6,7 and 8 , respectively for the multiphoton resonances of the ground state $\mid g>$ to the first excited state $1 e>$, to the second excited state $\mid 2 e>$ and to the fourth excited state $4 e>$. The third excited state $\mid 3 e>$ resembles a d-state and we find that the resonance on $|3 e>\leftrightarrow| g>$ involves a coupling three orders of magnitude smaller than those of $|1 e>\leftrightarrow| g>$ and $|4 e>\leftrightarrow| g>$. We emphasize that our calculations are beyond the dipole approximation so that the usual selection rules do not apply. In these three figures the solid line and dashed line represent the cases in which relativistic corrections due to the mass shift are ignored and included, respectively.

In figure 6 , we display the dominant resonant line which is located at $87.88 \omega$ following the calculation without the relativistic mass shift correction. This corresponds to the energy difference between the ground state and the first excited state including the conventional nonrelativistic Stark shift. When the leading relativistic correction to the electron kinetic energy is taken into account, we find from the dashed line in Fig 6 the corresponding resonance shifts to $87.45 \omega$, with $\omega$ denoting the laser frequency. The additional redshift of the resonance transition of $|1 e>\leftrightarrow| g>$ is as high as $0.43 \omega$ for the parameters chosen here. We refer to the correction as the "relativistic Stark shift". Furthermore, we note clear peaks displaced by two laser photons to both sides of the main resonanant lines; i.e. the laser stimulates the absorption or emission of two photons prior the emission of the high frequency photon from the resonant state. The effect of the relativistic Stark shift is clearly visible from the comparison of the dashed and solid line. In addition, small lines are visible which are displaced at about one-photon away from the resonances. Those would be forbidden under the dipole approximation, however appear because of quadric and high order contributions included in our calculation. Those are higher order terms and small in 
the weakly relativistic regime of interest here. Thus, higher order corrections to the position of this small peak as by the relativistic Stark shift are even smaller and are not visible for this peak displayed by one photon around the $|1 e>\leftrightarrow| g>$ transition. However, we note here already that it will be visible for higher excited states, for example for the $|4 e>\leftrightarrow| g>$ transition in figure 8.

For the resonance between $|2 e>\leftrightarrow| g>$, the result is plotted in figure 7 . It is similar to figure 6, but the relativistic Stark shift is only $0.39 \omega$. As addressed in section II(B), the second excited state $\mid 2 e>$ is symmetric in space and ressembles an s-state similar to the ground state $\mid g>$, so that the resonant transition $|2 e>\leftrightarrow| g>$ is considered. Thus only high order terms beyond the dipole term contribute. Considering the scale of the vertical axis, we find that the resonant signal is three orders of magnitude smaller than in the case for the $|1 e>\leftrightarrow| g>$ transition. In addition the relativistic Stark redshift turns out smaller than in the case of the $|1 e>\leftrightarrow| g>$ resonance, although the $\mid 2 e>$ state is not so tightly bound as the state $\mid 1 e>$. Moreover, the one-photon spaced peaks are no longer visible for the $|2 e>\leftrightarrow| g>$ transition. If we continue to explore the $|4 e>\leftrightarrow| g>$ resonance, we find that the relativistic Stark redshift becomes relatively large again, as indicated in Fig. 8. Since the state $\mid 4 e>$ is asymmetric and resembles a p-state as $\mid 1 e>$, the dipole transition $|4 e>\leftrightarrow| g>$ is permitted. Further the state $\mid 4 e>$ is less strongly bound by the ionic core and consequently much easier influenced by the external field. Therefore, the relativistic Stark shift attains $0.82 \omega$ and is thus larger than in the cases for the transitions $|1 e>\leftrightarrow| g>$ and $|2 e>\leftrightarrow| g>$. Finally the peaks spaced one-photon away from the resonance appear again as already in figure 6, however the relativistic Stark redshift becomes visible here also for these small peaks.

Figure 9 shows how the energy levels of the multiply charged ion of interest move under the radiation of an intense laser field. In the left part, the column (a) represents the situation without laser field and the central part (b) indicates the inclusion of the usual Stark shift but without relativistic corrections. The column (c) includes the "relativistic Stark shift" in addition to the usual Stark shift, which arises from the leading relativistic correction to 
the kinetic energy of the electron. The detailed position of the states $|1 e>| 2 e>$, and $\mid 4 e>$ is enlarged in the right part of figure 9. The series of transitions in columns (b) and (c) are corresponding to the solid lines and dotted lines in figures 6,7 and 8 , respectively. This figure gives us a clear picture of how the energy levels shift under an external intense laser field. The relativistic Stark shift, though small when compared to the absolute value of the energy levels, can clearly be identified in the radiation spectra, and should in principle be measurable in experiments.

\section{Spin effects}

We now turn to the role of the spin degree of freedom of the electron in intense laser-ion interaction. In first order in $v / c$ the Pauli equation already includes the coupling of the magnetic laser field to the spin as noted earlier [36]. We will not discuss this here and are more interested in the interaction of the spin degree of freedom with an operator describing the electron rather than the laser field. This is given by spin-orbit coupling arising first in second order in $v / c$.

With the laser intensity and ion charge increasing, such that second order effects become important, spin signatures in the dynamics are expected to be visible as noted first recently [23]. For free electrons, similar spin-induced forces have also been noted almost purely analyticaly, especially most interestingly a small one in the direction of the magnetic field of the laser field [26]. Here, we concentrate on the effect of the spin degree of freedom on bound electron dynamics and radiation in intense laser fields. In this case the used Hamiltonian $H_{F W}$ includes all second order terms in $1 / c^{2}$ as shown in equation (1), of which both the Pauli term $H_{P}$ and the spin-orbit coupling term contribute to spin effects. As noted in subsection A the Lorentz force may induce a significant angular motion around the ionic core with considerable orbital angular momentum $\mathbf{L}$ with respect to the origin set by the nucleus. We show that the resulting enhanced spin orbit coupling gives rise to observable effects in the electron dynamics and radiation. In particular we note a significant splitting 
of the non-symmetric bound states due to this additional interaction which leads to well separated doublets and four-line structures in the radiation spectra. In general terms we understand those also as indications that the influence of the spin and other relativistic effects are both principally observable in experiments and nonnegligible in theoretical treatments for relatively low laser intensities.

We are still interested in the weakly relativistic regime of optical laser intensities of up to at about $10^{17} \mathrm{Wcm}^{-2}$, which have been implemented in several laboratories worldwide and which still allows for laser - bound electron dynamics. We employ the 527nm (double Nd:glass) laser with an intensity of $7 \times 10^{16} \mathrm{~W} / \mathrm{cm}^{2}$ to interact with $\mathrm{Z}=12$ ions, which are intially spin-up polarized. The laser pulse has a 5.25-cycle turn-on and 100-cycle constant amplitude duration. In figure 10 we address the spin polarization itself and have displayed the expectation value of the electronic wavefunction in "spin-down" configuration as a function of the interaction time. We compare results from the full second order Hamiltonian $\mathbf{H}_{\text {FW }}$ including spin-orbit coupling with those where spin-orbit coupling $\mathbf{H}_{\text {so }}$ has been ignored. Both situations involve a spin flipping with twice the laser frequency. With spin-orbit coupling however the total flipping amplitude is higher because of a second oscillation due to the magnetic field of the frame of reference transformed nucleus $\mathbf{B}^{\prime}$. Finally the figure shows an effective polarization due to spin-orbit coupling in the turn-on phase, while without spin orbit coupling the electron periodically returns to the initial polarization in complete spin-up configuration.

The most significant qualitative features appear in the radiation spectrum in terms of strongly laser-enhanced line splitting. In figure 11 we have displayed the radiation spectrum of light emitted perpendicular to the plane spanned by the laser polarization and propagation directions and being polarized in $x$-direction. The upper row describes the situation governed by the Pauli Hamiltonian while the lower involves the full second order Hamiltoninan $\mathbf{H}_{\mathbf{F W}}$ in Eq.(1). Figures 11i(a), 11i(b) and 11i(c) show the spectral segments corresponding to the resonances of the first excited state $\mid 1 e>$ to the ground state $\mid g>$, the third excited state $\mid 3 e>$ with the ground state $\mid g>$, and the third excited state $\mid 3 e>$ to the first excited state 
$\mid 1 e>$. Comparing the upper and lower row we note shifts and splittings of the spectral components into a doublet in (a) and (b) and a four-line structure in (c). In addition, the relativistic Stark redshift is also observable, as discussed at length in III(B). We found also that the Darwin term due to $\mathbf{H}_{\mathbf{D}}$ has no notable effects in this situation. We confirmed that the spectral features displayed are generally well separated for ions with different charge states, should a possible experiment not allow for a pure sample of the ion of choice.

We explain the doublets and four-line structure with the splitting of the asymmetrical excited states $\mid 1 e>$ and $\mid 3 e>$ due to the additional spin orbit interaction as depicted in Fig. 11ii, while the symmetrical states, possibly s-states, remain unchanged. The splitting becomes larger with increasing laser intensity or charge of the ionic core. All transitions give rise to spectral features because the common selection rules do not apply in the parameter regime beyond the dipole approximation as investigated here. The bound states in Fig. 11ii drawn with thick lines indicate that those states of the split doublet are most populated explaining the relative heights of the spectral lines in Fig. 11i. The amount of population in the excited states is well above $1 \%$ (e.g. $1.25 \%$ in the first excited state) such that the total radiation should not be insignificant. We note that the amount of the splitting is $\frac{\Delta E}{\omega_{L}} \simeq 0.48$ for the state $\mid 1 e>$ and 0.04 for the state $\mid 3 e>$ (here, $\omega_{L}=0.0866$ a.u. is the applied laser frequency) so that the enhanced spin-orbit splitting should be easily measurable in experiments. Comparing those values with the amount of spin-orbit splitting without the presence of the laser field we have evaluated numerically via the same techniques $\frac{\Delta E_{0}}{\omega_{L}} \simeq 0.042$ for $\mid 1 e>$ and 0.004 for the state $\mid 3 e>$. Thus, we find that the total enhancement factor of the spin-orbit splitting due to the intense laser field for our set of parameters amounts to approximately $\simeq 10$. We note that those values should increase considerable for more intense laser fields and for higher charged ions. However, we also emphasize that for less charged ions, in particular hydrogen, spin orbit coupling has still little significance. 


\section{Generation of Coherent keV X-rays}

From nonrelativistic laser atom interaction it is well known that parts of the electronic wavepacket, for appropriate parameters, may tunnel through the Coulomb barrier, then propagate essentially freely in the laser field and when returning to the ionic core in the oscillating field may release their energy in form of radiation [8]. The corresponding radiation spectrum in terms of harmonics of the applied field is in fact quite attractive because quite high frequency coherent light is achievable. Instead of an exponential decay of the spectrum with respect to the harmonic order we here experience a plateau and a cut-off energy of the emitted harmonics at $I_{p}+3.17 U_{p}$. Here $I_{p}$ is the ionization potential of the ions and $3.17 U_{p}$ the maximal kinetic energy of the electron at the time of the return to the nucleus. This law is valid in the tunnel regime where the Keldysh parameter need fulfill $\gamma_{K}=\sqrt{\frac{I_{p}}{2 U_{p}}} \ll 1$.

The kinetic energy of electrons increases significantly with the laser intensity. Consequently higher harmonics are expected with an increasing laser intensity if the ionic potential is raising correspondingly, as shown recently already in the nonrelativistic regime with an output of more than 2000 low-frequency harmonics [37]. We here concentrate on the weakly relativitic regime and show that the harmonic order increases considerably by enhancing the charge of the ion and simultaneously increasing the laser field. Here we took special care on adapting the ratio of $U_{p}$ and $I_{p}$ such that we still remain in the tunneling regime.

In our calculations, we employ the $K r F$ laser (wavelength $\sim 248 n m$ ) to interact with multiply charged ions of charge $\mathrm{Z}=3$ and $\mathrm{Z}=4$. There are two advantages to use shortwavelength lasers: (1) the generated harmonics have a high efficiency and are well separated even near the cut-off because of $\omega_{2 n+1}-\omega_{2 n-1}=4 \pi / \lambda$ ( $\lambda$ being the fundamental laser wavelength); (2) the numerical resolution is better, as the box size is proportional to the square of laser wavelength and the CPU time to the cube. Moreover, the chosen laser wavelength is available in experiments. The pulse envelope is assumed to have a 10-cycle linear turn-on, followed by 10-cycles with constant maximal amplitude. For $\mathrm{Z}=3$ ions (potential parameters $\mathrm{k}=6.48, q_{e}=1.0$ ), we use the laser intensity of $2.5 \times 10^{16} \mathrm{~W} / \mathrm{cm}^{2}$. The result is shown in 
figure 12, in which we find as highest harmonic the 131st order. To our best knowledge, the maximum harmonic order ever obtained in experiments for this short driving wavelength is the 37th [10]. There the authors expressed that they believe that the $\mathrm{He}^{+}$ions may have also contributed to the harmonic emission. Our numerical simulation showns that this harmonic spectrum can be extended to the 131st order if the $Z=3$ ions $\left(L i^{2+}\right)$ are employed instead. This harmonic has a wavelength $\sim 1.9 \mathrm{~nm}$, and the efficiency indicated is still of the level $10^{-10} \sim 10^{-11}$ relative to the radiation at the fundamental frequency. Please note in figure 12 that we have only shown the radiation polarized in the laser polarization direction as the radiation polarized in the laser propagation direction is relatively small for the parameters chosen here.

Coherent X-rays even in the $\mathrm{keV}$ regime can be obtained when $Z=4$ ions (potential parameters $\mathrm{k}=10.7, q_{e}=1.0$ ) are used. In figure 13, we display the harmonic spectrum of $Z=4$ ions driven by a laser pulse with the same parameters as in the previous figure but with an intensity of $10^{17} \mathrm{~W} / \mathrm{cm}^{2}$. The spectrum polarized in the laser polarization direction in a) has been evaluated via the Fourier transform of $a_{x}(t)$, and the smaller contribution polarized in the laser propagation direction in b) via $a_{z}(t)$ in Eq. (10). The cut-off is enlarged as an insert in the right corner of figure $13 \mathrm{a}$ ). We emphasize that coherent radiation of the 427 th harmonic is clearly observable in spite of the low efficiency of $10^{-13}$ relative to the radiation at the fundamental frequency. The photon energy of this harmonic exceeds even $2 \mathrm{keV}$ and shall be useful for many applications, as e.g. for time-resolved X-ray diffraction. For the parameters chosen we find no significant deviation to the cut-off rule, even though the factor 3.17 is expected to alter with more than weakly relativitic free electron dynamics changing the kinetic energy at the recollision time. Most remarkably, and unfortunately, the plateau is tilting with increasing charge reducing the efficiency of the highest harmonics substantially. Thus our results indicate that a part of the electronic wave packet can still tunnel through the barrier formed by a deep ionic potential but the amount that tunnels out and returns reduces drastically with the ion charge. Thus with further increasing charge higher harmonic energies are possible but special care is devoted to the small efficiency. 
The calculations for figure 13 have been carried involving all first order terms in $v / c$, i.e. including the role of the magnetic field, and the leading terms in $(v / c)^{2}$. We have chosen parameters in the weakly relativistic regime such that higher terms will not make a difference in the results presented. We note that the spectrum in figure 13 a) in fact would look hardly different without all the terms in $v / c$, however the one in figure $13 \mathrm{~b}$ ) would hardly ressemble the correct one depicted. This is not surprising as the laser induced dynamics in the laser propagation direction is fully induced by the magnetic field component of the laser field.

Regarding experimental possibilities for high harmonics from multiply charged ions in the coherent keV X-rays, there are two ways at hand. There may be the double pulse experiment [38] where the first pulse is used to strip several or many electrons of the neutral atoms and then a moderately delayed pulse will interact with the obtained multiply charged ions and generate the coherent $\mathrm{keV}$ X-rays as described above. However, here the free electrons ionized by the first pulse may influence the phase matching of harmonic emissions and there may also be a mixture of many charge states. The alternative way is to shoot atoms through thin foils which is clearly a cleaner way to generate ions with a pure charge state [20]. Extremely high densities of multiply charged ions have been generated recently [39], which raises hope for a reasonable efficiency for ion charges well above those investigated here.

\section{E. High-order Above-threshold-ionization}

When laser field strengths are employed which are high with respect to the binding fields of the ionic core, a large part of the electron wavepacket will escape the vicinity of the ion and not return. Those electrons may in fact be quite energetic and will be of interest in this section.

We begin by calculating the photoelectron spectrum for $Z=3$ ions with the method described in section $\mathrm{II}(\mathrm{C})$. Here, we use a laser pulse with a 3-cycle turn-on, and 10-cycles with

constant maximal amplitude. The applied maximal intensity is equal to $2.5 \times 10^{16} \mathrm{~W} / \mathrm{cm}^{2}$, 
and the laser wavelength is $248 \mathrm{~nm}$ (corresponding to a photon energy near $5 \mathrm{eV}$ ). The resulting photoelectron spectrum is displayed in figure 14 . The spectral range from $860 \mathrm{eV}$ to $900 \mathrm{eV}$ is enlarged as an insert in the right-up corner of this figure. We note very energic electrons with energies up to $2 \mathrm{keV}$ with the usual photon-energy spacing characteristics of photoelectron peaks. Figure 15 shows the case for $Z=4$ ions with an appropriately higher laser intensity of $1.2 \times 10^{17} \mathrm{~W} / \mathrm{cm}^{2}$. As expected more energetic (above $5 \mathrm{keV}$ ) photoelectrons can be deteced. A rather regular spacing of the peaks in this above-threshold-ionization spectrum is still notable in this keV energy regime.

Thus next to extremely high harmonics we find also very energetic electrons due to above-threshold ionization. In more general terms those results and the ones in the previous section show that very high-order nonlinear effects are governing the interaction of multiply charged ions with very intense laser fields.

\section{CONCLUSION}

We believe to have shown that the physics of multiply charged ions in very intense laser fields is even richer than that for neutral atoms with moderately intense laser fields. There is not merely the effect of scaling the known effects for neutral atoms to the new intensity regime but the upcoming of many relativistic influences imposes a fundamentally different dynamics. We have seen that the magnetic field component of the laser field completely modifies the dynamics and may even induce a partially circular motion around the nucleus with the effect of a reduced expectation value just in the near vicinity of the ionic core. In the regime where second order terms in $v / c$ are important, the relativistic mass shift modifies clearly the positions of the spectral components in the multiphoton regime. Amplification was found on resonances involving of the order of 100 photons with interesting non-dipole spectral features displaced few harmonics away from the atomic resonances. The spin, usually of no importance in the nonrelativistic regime, starts to oscillate and via spin-orbit coupling modifies substantially dynamics and radiation. The laser enhanced splitting of 
resonant spectral lines is a clear relativistic quantum signature.

Those aspects are associated with fundamentally new influences in the weakly relativistic regime with respect to the nonrelativistic case and were most conveniently described by applying the expansion of the Dirac equation up to the second order in $v / c$. This allowed us to relate each effect to different parts of the Hamiltonian and to carry out our numerical investigations even for the low frequencies available in most present day high power laser systems.

We stressed also that the combination of highly charged ions and high power lasers can be useful for applications. High-order above threshold ionization was shown to give rise to photoelectrons in the multi-keV regime already for laser intensities around $10^{17} \mathrm{~W} / \mathrm{cm}^{2}$. More interestingly parts of those highly energetic electron wavepackets may return to the nucleus and we indicated also coherent high harmonics in the keV regime. Even though for high harmonic generation towards the coherent hard X-ray regime the quantitative aspect appears most attractive, we pointed out also qualitative changes as the problematic titling of the plateau of the harmonic spectrum with increasing ion charge.

The authors acknowledge funding from the German Research Foundation (Nachwuchsgruppe within Sonderforschungsbereich 276). SXH would like to thank W. Becker for fruitful discussions and acknowledges present funding from the Alexander von Humboldt Foundation. CHK acknowledges helpful discussions with M. Casu, J. Ullrich and M. W. Walser.

\footnotetext{
* Present address: Max-Born-Institut for nonlinear Optics and Short Pulse Spectroscopy, Rudower Chaussee 6, 12489 Berlin, Germany. Present email: suxinghu@mbi-berlin.de

** Email address: keitel@physik.uni-freiburg.de
} 


\section{REFERENCES}

[1] D. Strickland and G. Mourou, Opt. Commun. 56, 219 (1985); P. Maine, et al, IEEE J. Quantum Electron, 24, 398 (1988).

[2] K. Yamakawa, H. Shiraga, Y. Kato, Opt. Lett. 16, 1593, (1991); C. Rouyer, et al, ibid 18, 214 (1993); J. P. Chambaret, et al, ibid 21, 1921 (1996); C. P. J. Barty, et al, ibid, 21, 668, (1996); M. D. Perry, et al, ibid, Opt. Lett. 24160 (1999).

[3] M. Protopapas, C. H. Keitel, and P. L. Knight, Rep. Progr. Phys. 60, 389 (1997); Atoms in Intense Laser Fields, ed. by M. Gavrila (Academic, San Diego, 1992); H. R. Reiss, Prog. Quant. Electr. 16, 1 (1992); C. J. Joachain, M. Dörr and N. Kylstra, Adv. At. Mol. Phys. 42, 225 (2000).

[4] A. D. Bandrauk, Molecules in Laser Fields, (Dekker, New York, 1993).

[5] see for example, A. McPherson, et al Nature(London) 370, 631 (1994); Phys. Rev. Lett. 72, 1810 (1994); T. Ditmire, et al, Phys. Rev. Lett. 75, 3122 (1995) ibid. Nature (London) 386, 54 (1997) and 398, 489 (1999).

[6] M. D. Perry and G. Mourou, Science 264, 917 (1994); P. A. Norreys, et al, Phys. Rev. Lett. 76, 1832 (1996).

[7] B. W. Shore and P. L. Knight, J. Phys. B 20, 413 (1987); W. Becker, S. Long and J. McIver, Phys. Rev. A41 , 4112 (1990) and A50, 1540 (1994); A. L'Huillier and P. Balcou, Phys. Rev. Lett. 70, 744 (1993); J. J. Macklin, J. D. Kmetec, C. L. Gordon III, and S. E. Harris, Phys. Rev. Lett. 70, 766 (1993); S.G. Preston et al, Phys. Rev. A53, R31 (1996).

[8] P. B. Corkum, Phys. Rev. Lett. 71, 1994 (1993); J. L. Krause, K. J. Schafer, K. C. Kulander, Phys. Rev. Lett. 68, 3535 (1992); M. Lewenstein, et al, Phys. Rev. A 49, 2117 (1994).

[9] Ch. Spielmann, et al, Science 278, 661 (1997); M. Schnürer, et al, Phys. Rev. Lett. 80, 
3236 (1998); Ch. Spielmann, et al, IEEE 4, 249 (1998); G. Tempea, M. Geissler and T. Brabec, J. Opt. Am B16, 669 (1999); G. Tempea et al, Phys. Rev. Lett. to be published (2000); M. W. Walser, T. Brabec and C.H. Keitel (in preparation).

[10] Z. Chang, et al, Phys. Rev. Lett. 79, 2967 (1997); H. Kapteyn and M. Murnane, Phys. World 12, 31 (1999).

[11] P. Agostini, et al, Phys. Rev. Lett. 42, 1127 (1979); R. R. Freemann, et al, Phys. Rev. Lett. 59, 1092 (1987).

[12] Q. Su, J. H. Eberly, and J. Javanainen, Phys. Rev. Lett. 64, 861 (1990); V. C. Reed, P. L. Knight and K. Burnett, Phys. Rev. Lett. 671415 (1993).

[13] J. Grochmalicki, M. Lewenstein and K. Rzazewski, Phys. Rev. Lett 66, 1038 (1991); T. Katsouleas and W.B. Mori, Phys. Rev. Lett. 70, C1561 (1993); C. H. Keitel, P. L. Knight, Phys. Rev. 51, 1420 (1995); J. R. Vázquez de Aldana and Luis Roso. Opt. Expr. 5, 144 (1999); V. P. Krainov, J. Phys. B 32, 1607 (1999); R. M. Potvliege, Las. Phys. 10, 143 (2000); H. R. Reiss, to be published (2000); N. J. Kylstra et al, to be published (2000).

[14] C. H. Keitel, P. L. Knight, and K. Burnett, Europhys. Lett. 24, 539 (1993); N. J. Kylstra, A. M. Ermolaev, and C. J. Joachain, J. Phys. B 30, L449 (1997); C. H. Keitel et al, J. Phys. B 31, L75 (1998); C. Szymanowski, C. H. Keitel and A. Maquet, Las. Phys. 9, 133 (1999); J. C. Csesznegi, G.H. Rutherford, Q. Su nad R. Grobe, Las. Phys. 9, 41 (1999); R.E. Wagner, R.J. Reverly, Q, Su and R. Grobe, Phys. Rev. A 60, 5402 (1999).

[15] U.W. Rathe, C.H. Keitel, M. Protopapas and P.L. Knight, J. Phys. B 30, L531 (1997); C. Szymanowski et al Phys. Rev. A 56, 3846 (1997); J.W. Braun, Q. Su and R. Grobe, Phys. Rev. A 59,604 (1999).

[16] K. W. D. Ledingham, et al, Phys. Rev. Lett. 84, 899 (2000); G. Pretzler et al., Phys. 
Rev. E 58, 1165 (1998); T. E. Cowan, et al, Phys. Rev. Lett. 84, 903 (2000).

[17] C.I. Moore, J. P. Knauer, D. D. Meyerhofer, Phys. Rev. Lett. 77, 2335 (1996); S. J. McNaught, J. P. Knauer and D. D. Meyerhofer, Phys. Rev. A58, 1399 (1998); C. Bula et al, Phys. Rev. Lett 76, 3116 (1996); S.Y. Chen, A. Maksimchuck and D. Umstadter, Nature (London) 396, 653 (1998).

[18] L.S. Brown and T.W. Kibble, Phys. Rev. 133, A705 (1964); E.S. Sarachik and G.T. Schappert, Phys. Rev. D 1, 2738 (1970) ; A.I. Nikishov, V.I. Ritus, Sov. Phys. JETP 19, 529 (1964); I.I. Goldman, Sov. Phys. JETP 19, 954(1964).

[19] D. J. Urbach and C. H. Keitel, Phys. Rev. A 61043409 (2000).

[20] P.H. Kokler and Th. Stoehlker, Adv. At. Mol. Opt. Phys. 37, 297 (1996); J. Ullrich et al, J. Phys. B 30, 2917 (1997); J.R. Crespo López-Urrutia et al, Verhandl. Deutsch. Phys. Gesell., 928 (2000).

[21] E. M. Snyder, et al, Phys. Rev. Lett. 77, 3347 (1996); T. Ditmire et al., Phys. Rev. Lett. 78, 2732 (1997); Phys. Rev. A 57, 369 (1998) .

[22] S. X. Hu, C. H. Keitel, Europhys. Lett. 47, 318 (1999).

[23] S. X. Hu, C. H. Keitel, Phys. Rev. Lett. 83, 4709 (1999).

[24] L. L. Foldy and S. A. Wouthuysen, Phys. Rev. 78, 29 (1950).

[25] Paul Strange, Relativistic Quantum Mechanics, (Cambridge University Press, Cambridge, 1998).

[26] M. W. Walser and C. H. Keitel, J. Phys. B: At. Mol. Opt. Phys. 33, L221 (2000); M. W. Walser, C. Szymanowski and C. H. Keitel, Europhys. Lett. 48, 533 (1999).

[27] One atomic mass unit $=m_{e}=9.1094 \times 10^{-31} \mathrm{~kg}$; one atomic charge unit $=e=1.6022 \times$ $10^{-19} \mathrm{C}$; one atomic action unit $=\hbar=1.0546 \times 10^{-34} \mathbf{J}$ s; one atomic length unit (Bohr radius $)=0.5292 \times 10^{-10} \mathrm{~m}$; one atomic energy unit $=27.2112 \mathrm{eV}$; one atomic field strength 
unit $=5.1422 \times 10^{9} \mathrm{~V} / \mathrm{cm}$; one atomic time unit $=0.0242 \mathrm{fs} ;$ one atomic frequency unit $=4.1341 \times 10^{16} \mathrm{~Hz}$; one atomic angular frequency unit $=2.5975 \times 10^{17} \mathrm{rad} / \mathrm{s}$, it is corresponding to the atomic wavelength unit $=45.616 \mathrm{~nm}$; one atomic intensity unit $=3.5095 \times 10^{16} \mathrm{~W} / \mathrm{cm}^{2}$, the corresponding field amplitude can be evaluated by $E($ au. $)=0.5338 \times 10^{-8} \times \sqrt{I\left(\mathrm{~W} / \mathrm{cm}^{2}\right)}$.

[28] K. C. Kulander, et al, in Proceedings of the Workshop on Super-intense Laser Atom Physics (SILAP), ed. by B. Piraux, (Plenum, New York, 1993).

[29] J. H. Eberly, Q. Su and J. Javanainen, Phys.Rev.Lett. 62881 (1989).

[30] M. R. Hermann and J. A. Fleck, Jr., Phys. Rev. A 38, 6000 (1988); S. X. Hu, Z. Z. Xu, Phys. Rev. A56, 3916 (1997); S. X. Hu, W. X. Qu, Z. Z. Xu, Phys. Rev. A 57, 3770 (1998).

[31] M. D. Feit, J. A. Fleck, Jr., and A. Steiger, J. Comput. Phys. 47, 412 (1982).

[32] J. L. Krause, K. J. Schafer, and K. C. Kulander, Phys. Rev. A 45, 4998 (1992); S. X. Hu, W. X. Qu, Z. Z. Xu, J. Phys. B 31, 1523 (1998).

[33] R. W. Heather, Comput. Phys. Commun. 63, 446 (1991); S. X. Hu, W. X. Qu, Z. Z. Xu, Appl. Phys. Lett. 73, 2552 (1998).

[34] M. Protopapas, C. H. Keitel, and P. L. Knight, J. Phys. B 29, 591 (1996).

[35] R. Taïeb, V. Véniard and A. Maquet, Phys.Rev.Lett. 81, 2882 (1998).

[36] O. Latinne, C. J. Joachain, and M. Dörr, Europhys. Lett. 26, 333 (1994); J. R. Vázquez de Aldana and Luis Roso, (to be published).

[37] M. Casu, C. Szymanowski, S. X. Hu and C. H. Keitel, (to be published).

[38] for example, J. Zhang, et al, Science Vol276, 1097 (1997).

[39] S. Debosh et al., JETP (Rus.) 115, 2051 (1999); G.V. Ivanenkov et al., JETP (Rus.)114, 
1216 (1998). 


\section{FIGURES}

FIG. 1. Schematic scetch relating near-optical laser intensities to the charge state $Z$ of a single electron ion such that the laser electric field and that of the nucleus become comparable, i.e. the laser field induces a nonperturbative interaction without large ionization. The emphasis of this paper is on the weakly relativistic regime from $Z=3$ to $Z=12$

FIG. 2. 2.a) The energy-level structure of a model multiply charged ion with $\mathrm{Z}=12$. The potential is described by a soft-core model $-k / \sqrt{q_{e}+x^{2}+z^{2}}$ with $\mathrm{k}=80.32$ and $q_{e}=1.0$ and ground state energy of -72au.. 2.b) The probability distributions of the electronic wavepackets for our model multiply charged ion for the three lowest energy states $|g>| 1 e>$, and $\mid 2 e>$.

FIG. 3. The center-of-mass evolution of the electronic wave packet for the cases with B-field ("full circles") and without B-field ("open diamonds"). The laser parameters involve a wavelength of $248 \mathrm{~nm}$, an intensity of $1,2 \times 10^{17} \mathrm{~W} / \mathrm{cm}^{2}$, a three-cycle linear turn-on and a ten-cycle constant amplitude duration. For $L i^{2+}$ in a) $\left(\mathrm{Z}=3, q_{e}=1.0, k=6.48\right)$ there is a significant drift in the laser propagation direction and in addition to substantial ionization (not visible) a considerable part of the wavepacket returns to the nucleus opposite the laser propagation direction. A reduction of the expectation value of the electronic wave packet is observed around the close vicinity of the ionic core for $B e^{3+}\left(\mathrm{Z}=4, q_{e}=1, \mathrm{k}=10.7\right)$ as indicated by the arrow in $\left.\mathrm{b}\right)$, when the B-field is considered.

FIG. 4. a.) The relaxation radiation spectrum of the hollow ion $\left(B e^{3+}\right.$ as prepared for fig. 3b) during the time of thirty laser cycles after the laser pulse. The strongly reduced expectation value close to the nucleus of the active electron is reversed again and x-ray radiation is emitted. The corresponding transitions are plotted in b), which are modified by the Stark shift. 
FIG. 5. The spectrum of $\mathrm{Z}=12$ model ions (with level structure indicated by Fig.2), which are exposed to an intense laser pulse of intensity $7 \times 10^{16} \mathrm{~W} / \mathrm{cm}^{2}$ and the laser wavelength $527 \mathrm{~nm}$. The laser pulse has a 5.25-cycle turn-on, followed by a 100-cycle period with constant amplitude. The laser parameters are in the multiphoton regime with respect to the ion charge so that resonant structures are visible. The solid line and the dotted lines represent the cases without and with the leading relativistic correction, respectively.

FIG. 6. The segment of Fig.5 for the multiphoton resonant transition between the bound states $|1 e>\leftrightarrow| g>$. The solid and dotted lines represent the cases without and with the leading relativistic correction, respectively. A clear redshift of $0.43 \omega$ of the resonant line is observed when the leading relativistic correction is taken into account. It can be attributed to the relativistic Stark shift of the energy levels of the ion.

FIG. 7. Same as Fig.6 but for the resonant transition $|2 e>\leftrightarrow| g>$. The redshift is up to $0.39 \omega$ for this case.

FIG. 8. Same as Fig.6 but for the resonant transition $|4 e>\leftrightarrow| g>$. A large redshift of $0.82 \omega$ for this resonant transition is visible .

FIG. 9. Energy level diagram of the four lowest eigenstates of the $\mathrm{Z}=12$ ion in intense laser field. In the left part, the column (a) shows the eigenstates without external fields, column (b) involves the conventional Stark shift and the relativistic Stark shift is included in column (c). The transitions in columns (b) and (c) are corresponding to the solid and dotted lines in figures 6, 7 and 8 . The details of the shifting process are enlarged in the right part for states $|1 e>| 2 e>$, and $\mid 4 e>$. 
FIG. 10. The dynamics of the spin degree of freedom for the situations without (a) and with (b) spin-orbit interaction as viewed from the population of the wavefunction with spin-down polarization. The initial electron is spin-up polarized. The intense laser-enhanced spin-orbit coupling leads to an effective spin polarization in the turn-on phase and an additional oscillation due to magnetic field from the nucleus in the rest frame of the electron. The laser parameters involve a wavelength of $527 \mathrm{~nm}$, an intensity of $7 \times 10^{16} \mathrm{~W} / \mathrm{cm}^{2}$, a 5.25 -cycle linear turn-on and a 10-cycle duration with constant amplitude. The parameters for the ionic core are $q_{e}=1, \mathrm{k}=80.32(\mathrm{Z}=12$ ions).

FIG. 11. i). Radiation spectrum of the laser driven $\mathrm{Z}=12$ ion. The first row corresponds to the Pauli modelled system, and the second row is for the case where spin-orbit coupling including the relativistic mass shift and Zitterbewegung is taken into account. Figures (a), (b) and (c) are associated, respectively, with transitions from $\mid 1 e>$ to $|g>| 3 e>$, to $\mid g>$, and $\mid 3 e>$ to $\mid 1 e>$ (see also Fig.11.ii). The spectral lines split into doublets ((a) and (b)) and a four-line structure (c) due to the spin-orbit interaction. All parameters are same as those in figure 10 part from 100 cycles at full strength rather than 10;

ii). The schematic diagram of state-splitting as induced by the enhanced spin-orbit interaction due to the intense laser field. We note that asymmetric states split as opposed to symmetric states. Transitions (a), (b) and (c) are associated with the corresponding spectral lines in figure 11.i).

FIG. 12. The harmonic spectrum emitted from $\mathrm{Z}=3$ ions with parameters $\mathrm{k}=6.48$ and $q_{e}=1.0$ characterizing the potential. The laser intensity is $2.5 \times 10^{16} \mathrm{~W} / \mathrm{cm}^{2}$, and the $K r F$ laser wavelength with $248 \mathrm{~nm}$ is applied. The laser pulse has a linear 10-cycle turn-on and is followed by a 10-cycle duration with constant amplitude. The 131st harmonic is visible in the the cut-off frequency. 
FIG. 13. Same as Fig.12 but for $\mathrm{Z}=4$ ions $\left(\mathrm{k}=10.7, q_{e}=1.0\right)$. The laser intensity is further increased to $10^{17} \mathrm{~W} / \mathrm{cm}^{2}$ resulting in harmonic emission up to the 427th order with a photon energy well above $2 \mathrm{keV}$. Consequently coherent keV X-rays can be obtained through high harmonic generation with multiply charged ions. The contribution of the harmonic yield in the laser polarization direction is depicted in a) and the one in the laser propagation direction in b).

FIG. 14. The photoelectron spectrum for $\mathrm{Z}=3$ ions. The laser parameters are the same as those in Fig.12 but with a steeper turn-on of 3 cycles. We can observe very energetic (2keV) photoelectrons and the ATI peaks are spaced by the photon energy even at electron energies close to the order of $1 \mathrm{keV}$.

FIG. 15. Similar to Fig.13 but for $\mathrm{Z}=4$ ions. The laser parameters are the same as those in Fig. 14 but involving a the higher intensity of $1.2 \times 10^{17} \mathrm{~W} / \mathrm{cm}^{2}$. More energetic $\sim 5 \mathrm{keV}$ photoelectrons are ejected, while conventional ATI features are still conserved. 


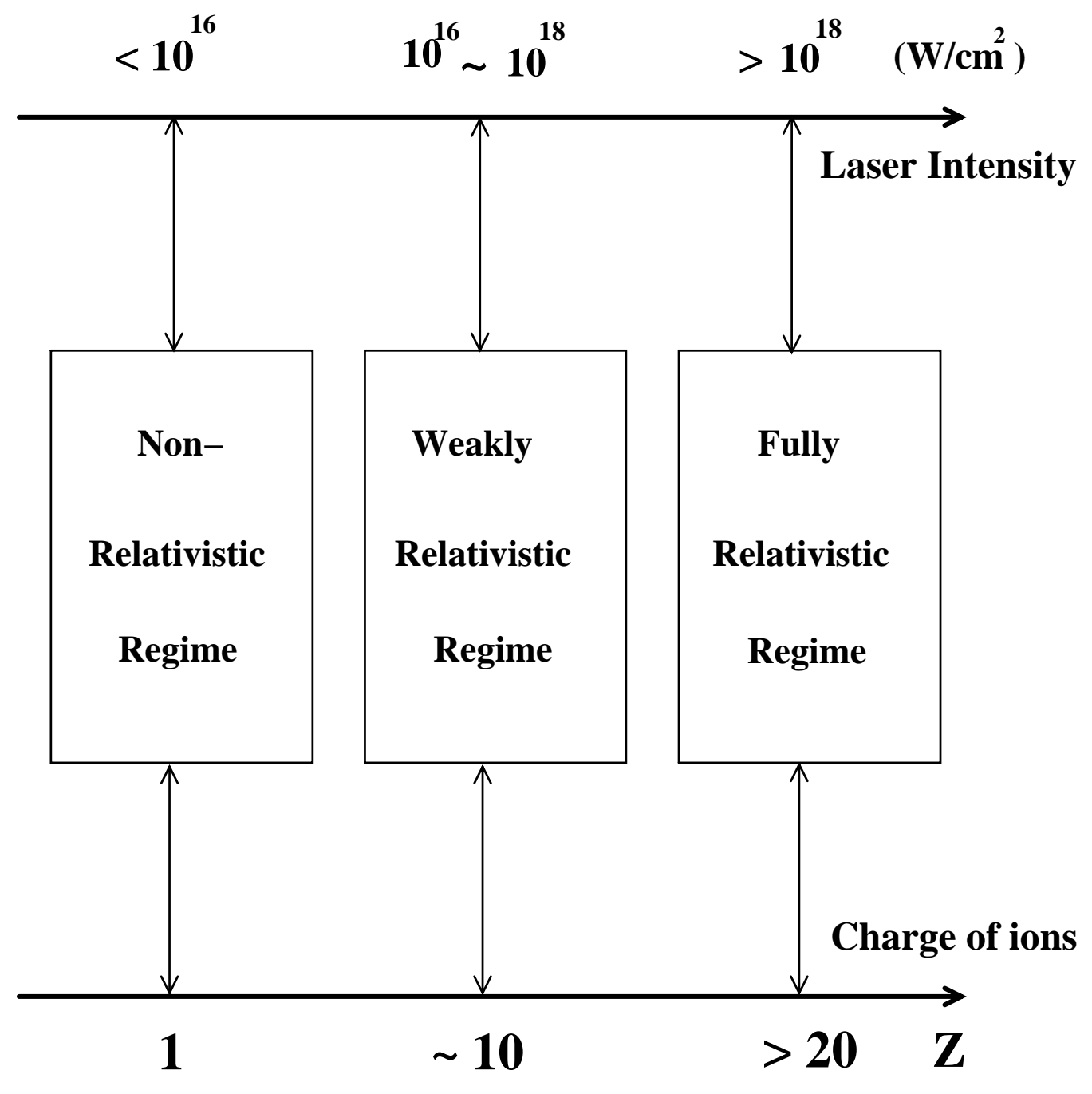

Fig. 1: S. X. Hu and C. H. Keitel, "Dynamics of ..." 


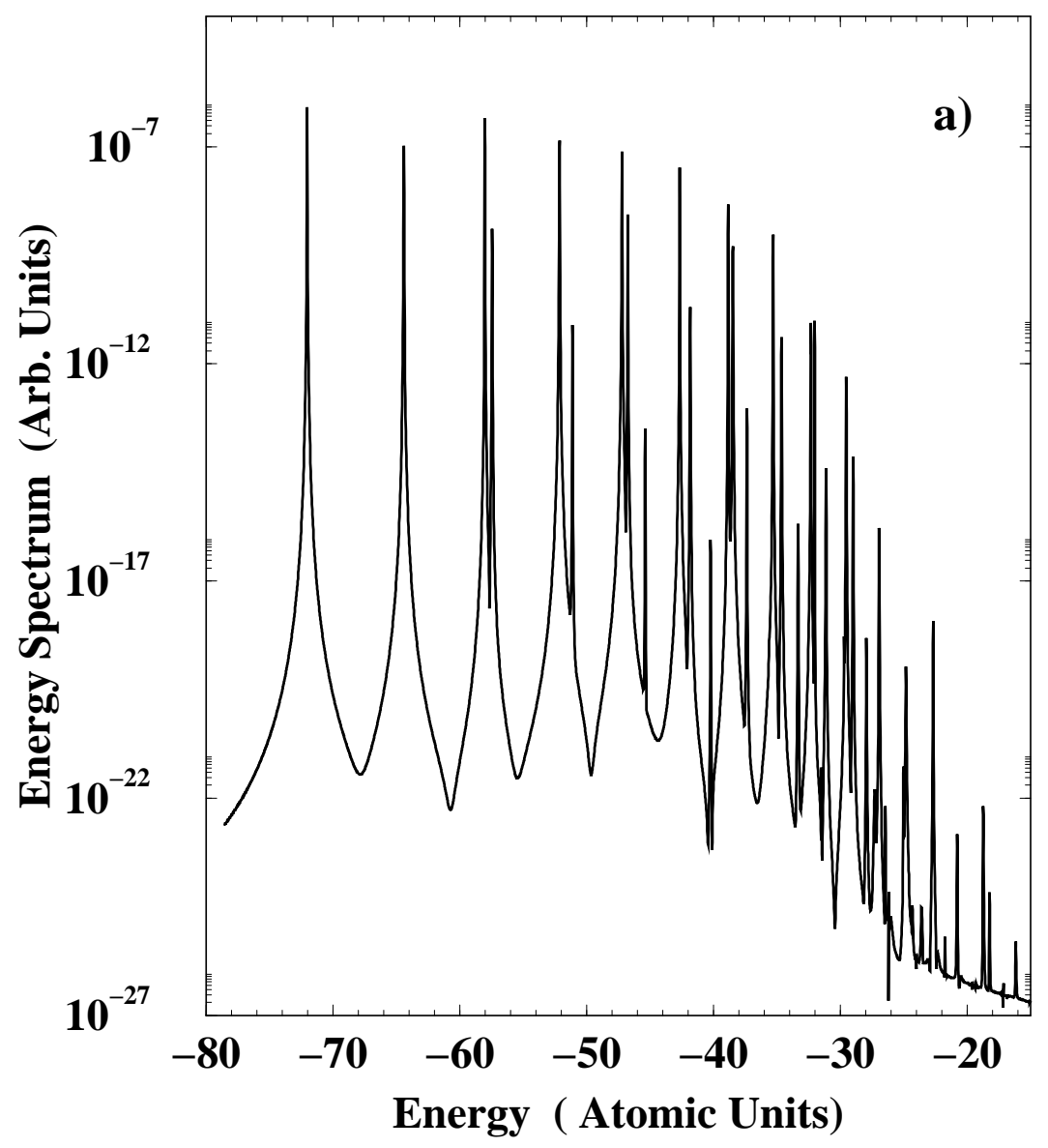

Fig. 2a: S. X. Hu and C. H. Keitel, "Dynamics of ..." 
b)
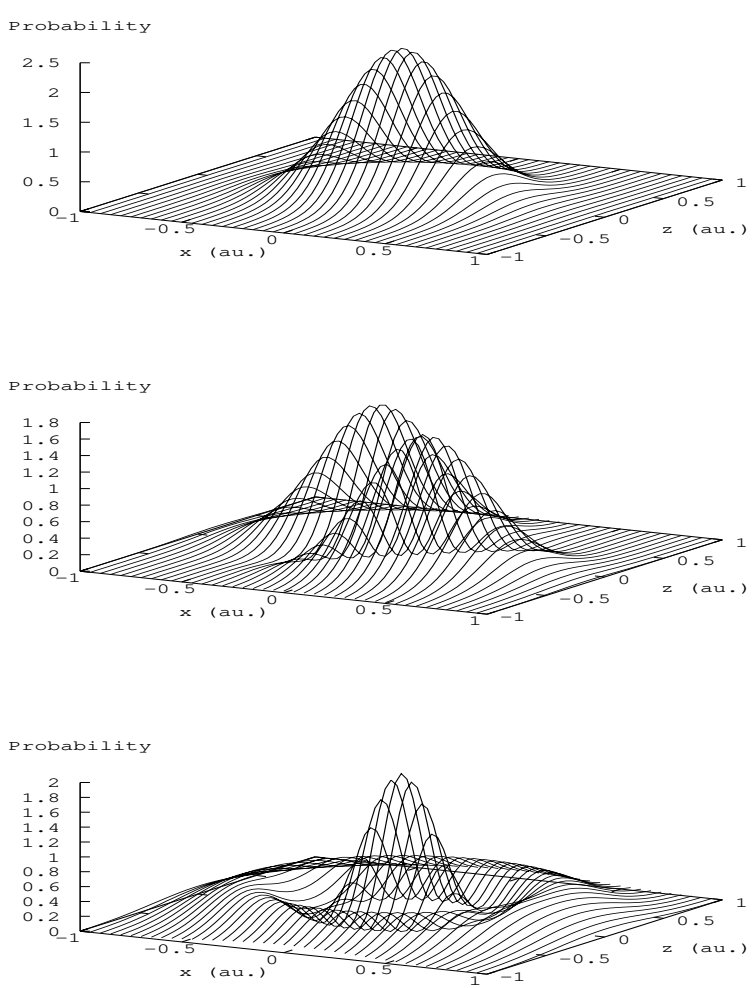

Fig. 2b: S. X. Hu and C. H. Keitel, "Dynamics of ..." 


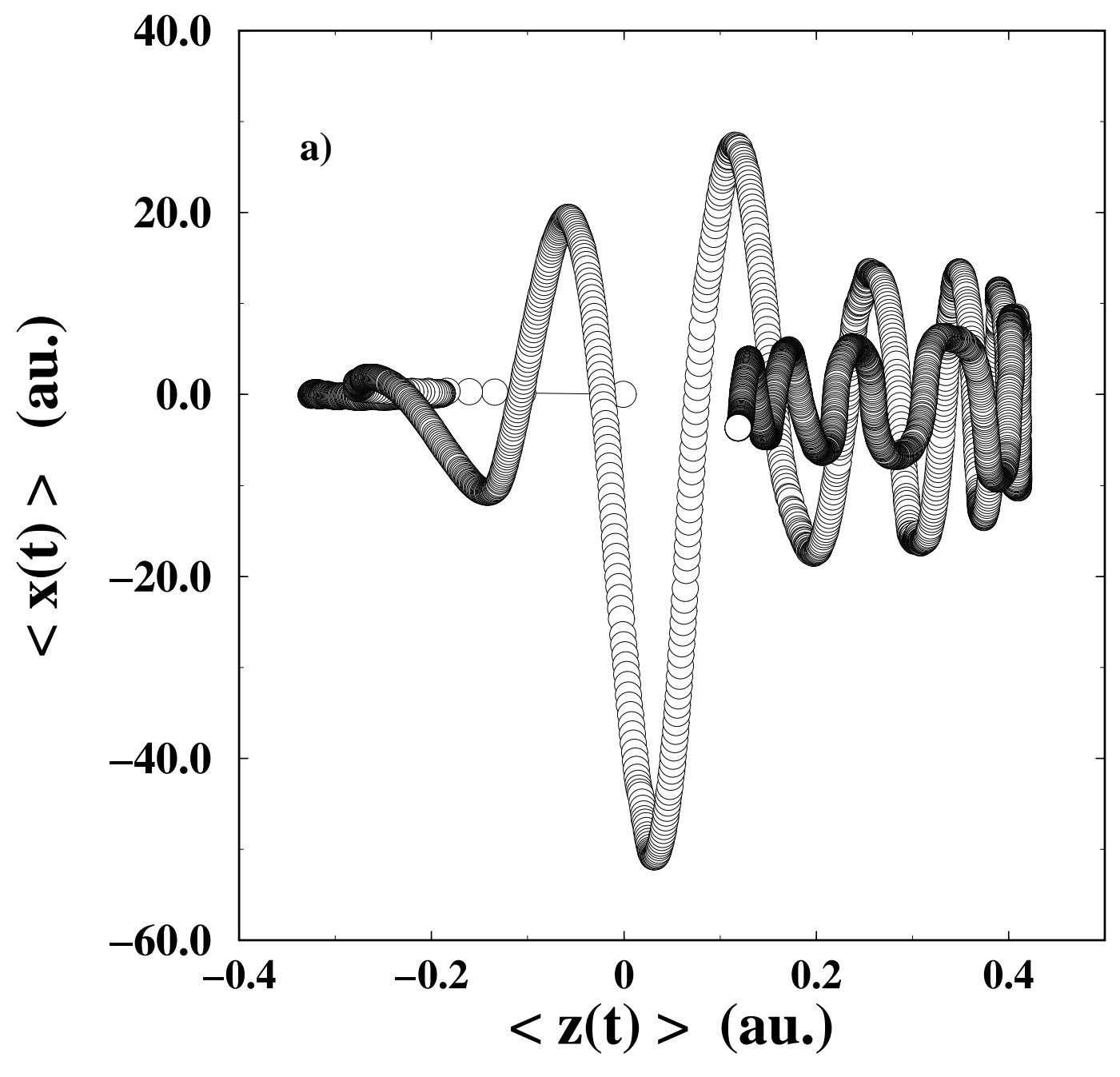

Fig. 3a: S. X. Hu and C. H. Keitel, "Dynamics of ..." 


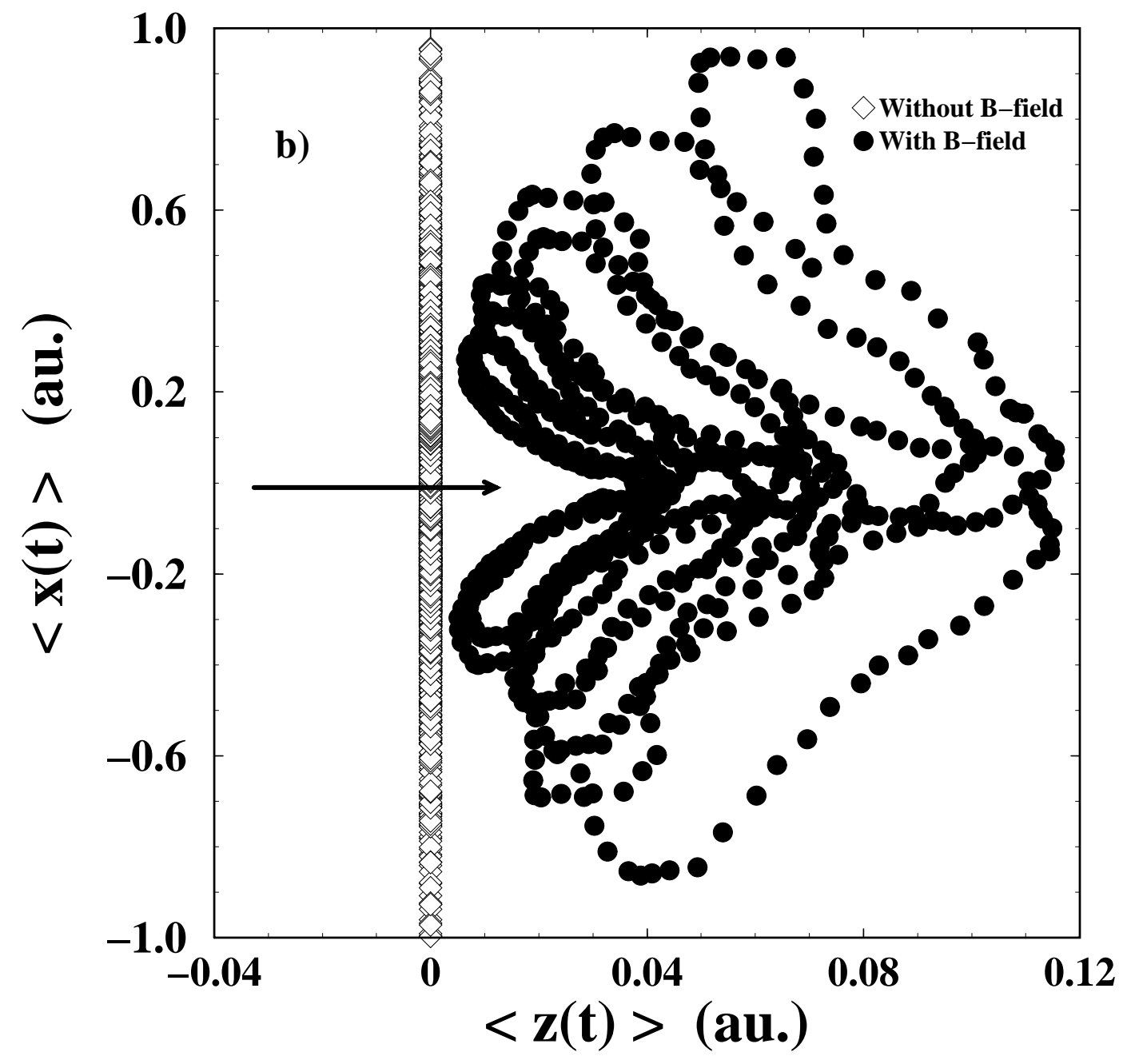

Fig. 3b: S. X. Hu and C. H. Keitel, "Dynamics of ..." 

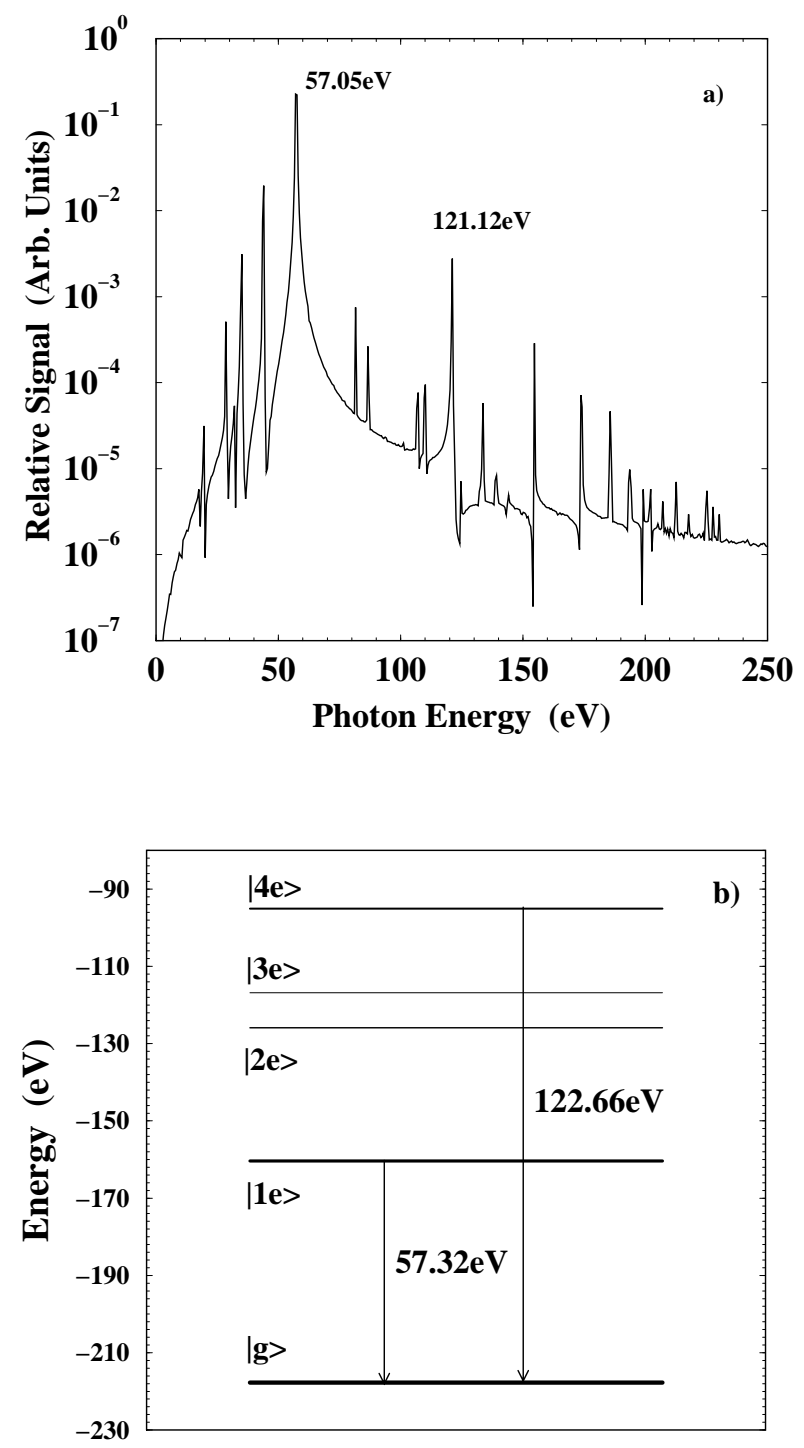

Fig. 4: S. X. Hu and C. H. Keitel, "Dynamics of ..." 

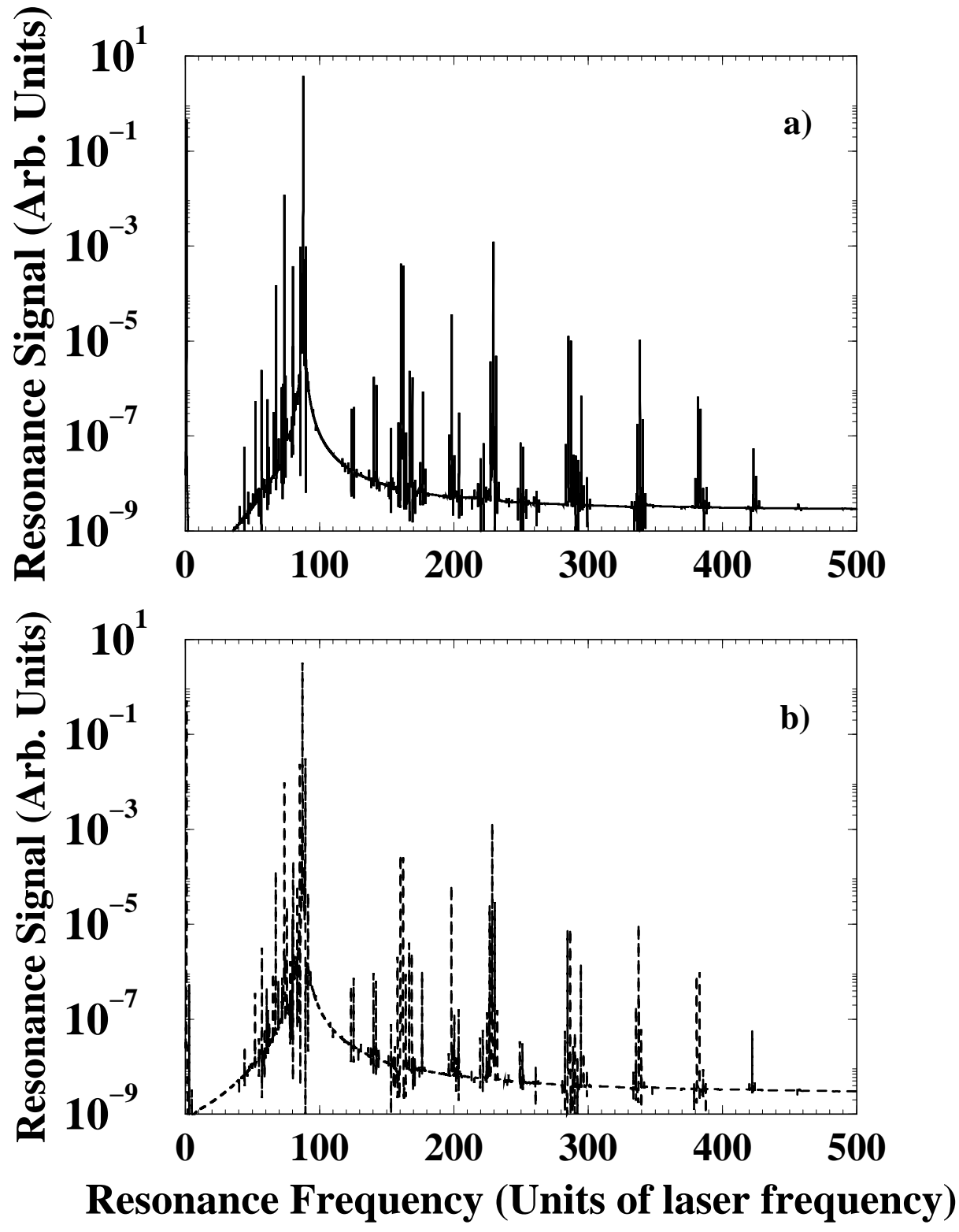

Fig. 5: S. X. Hu and C. H. Keitel, "Dynamics of ..." 


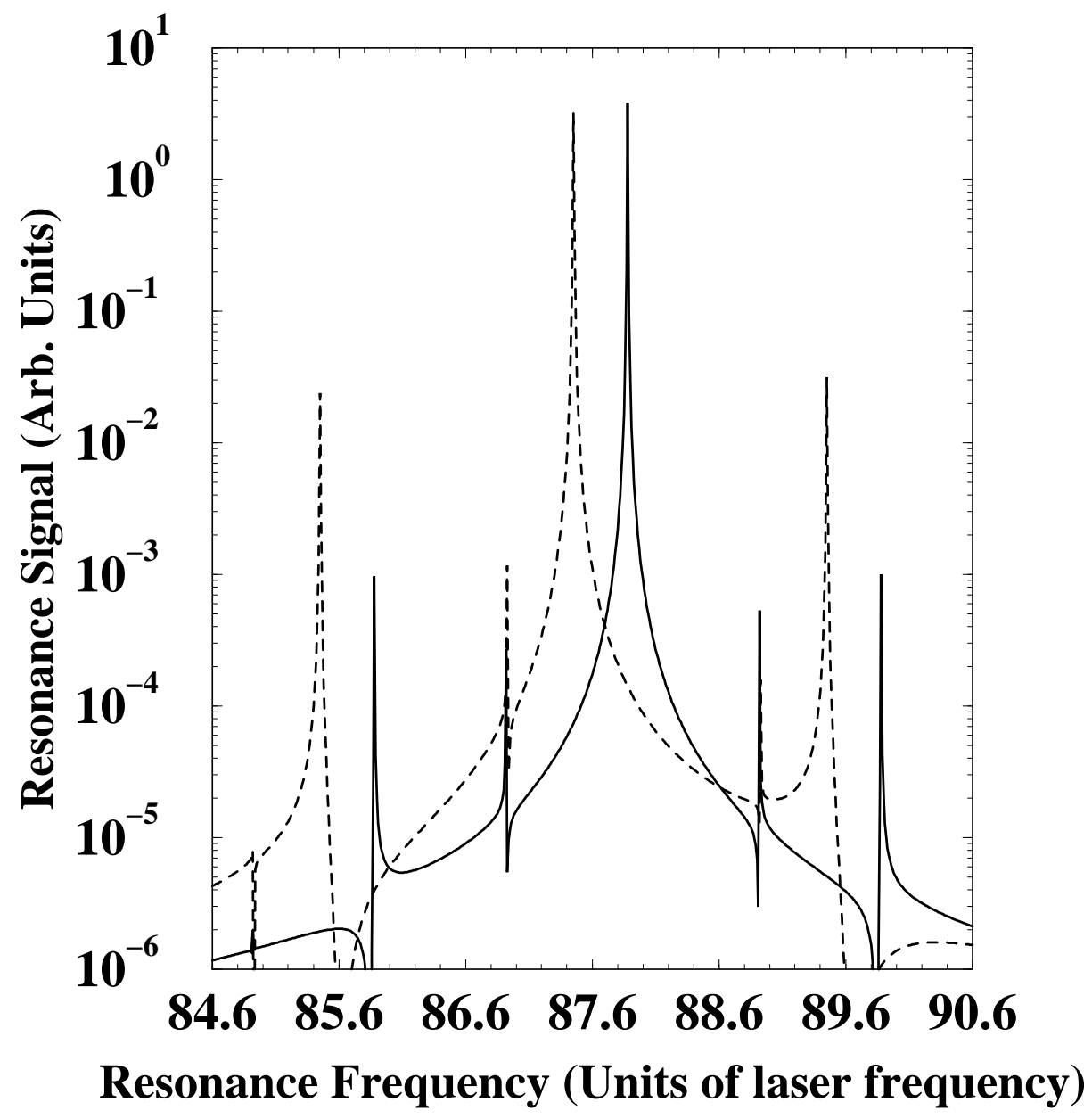

Fig. 6: S. X. Hu and C. H. Keitel, "Dynamics of ..." 


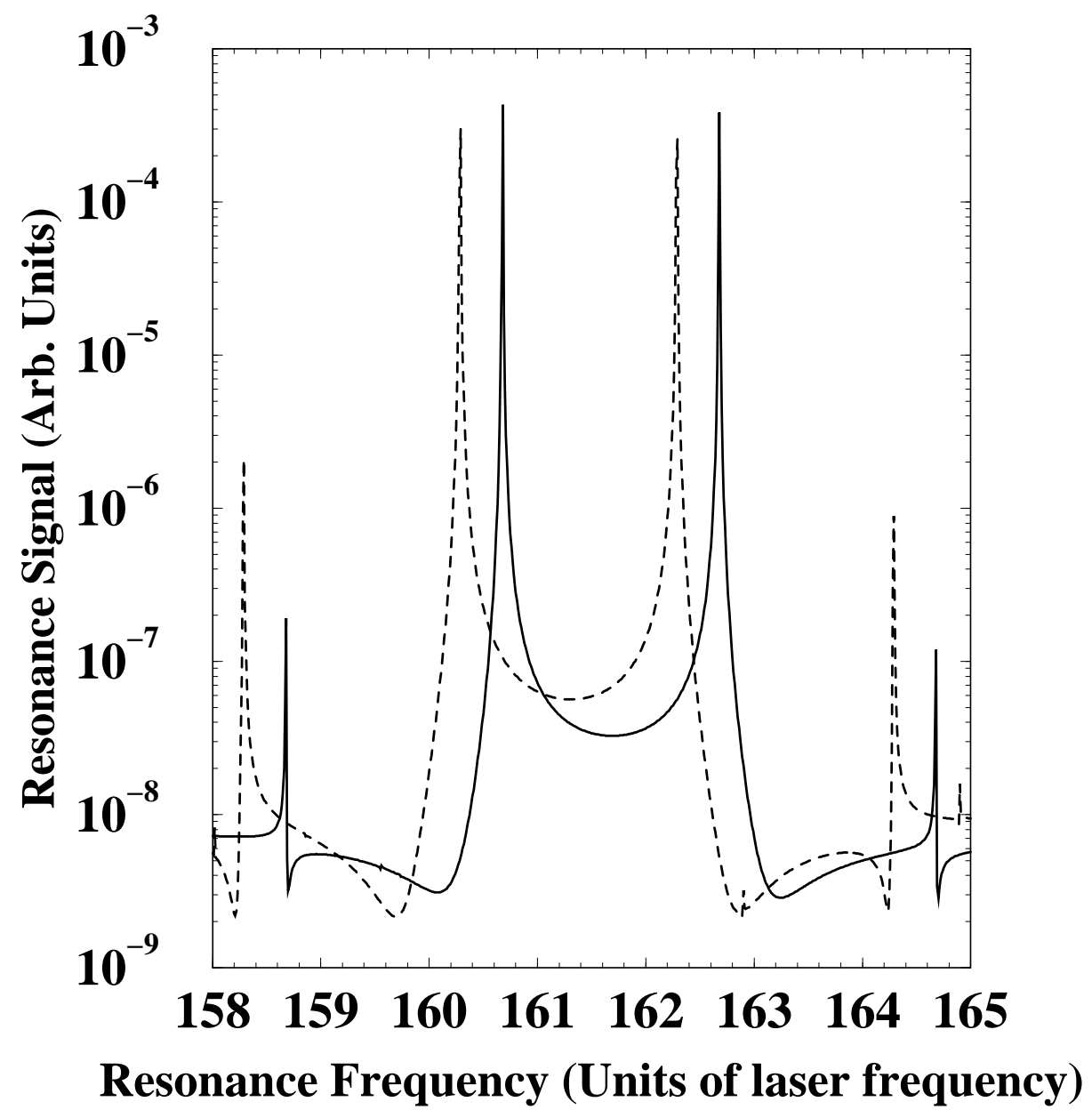

Fig. 7: S. X. Hu and C. H. Keitel, "Dynamics of ..." 


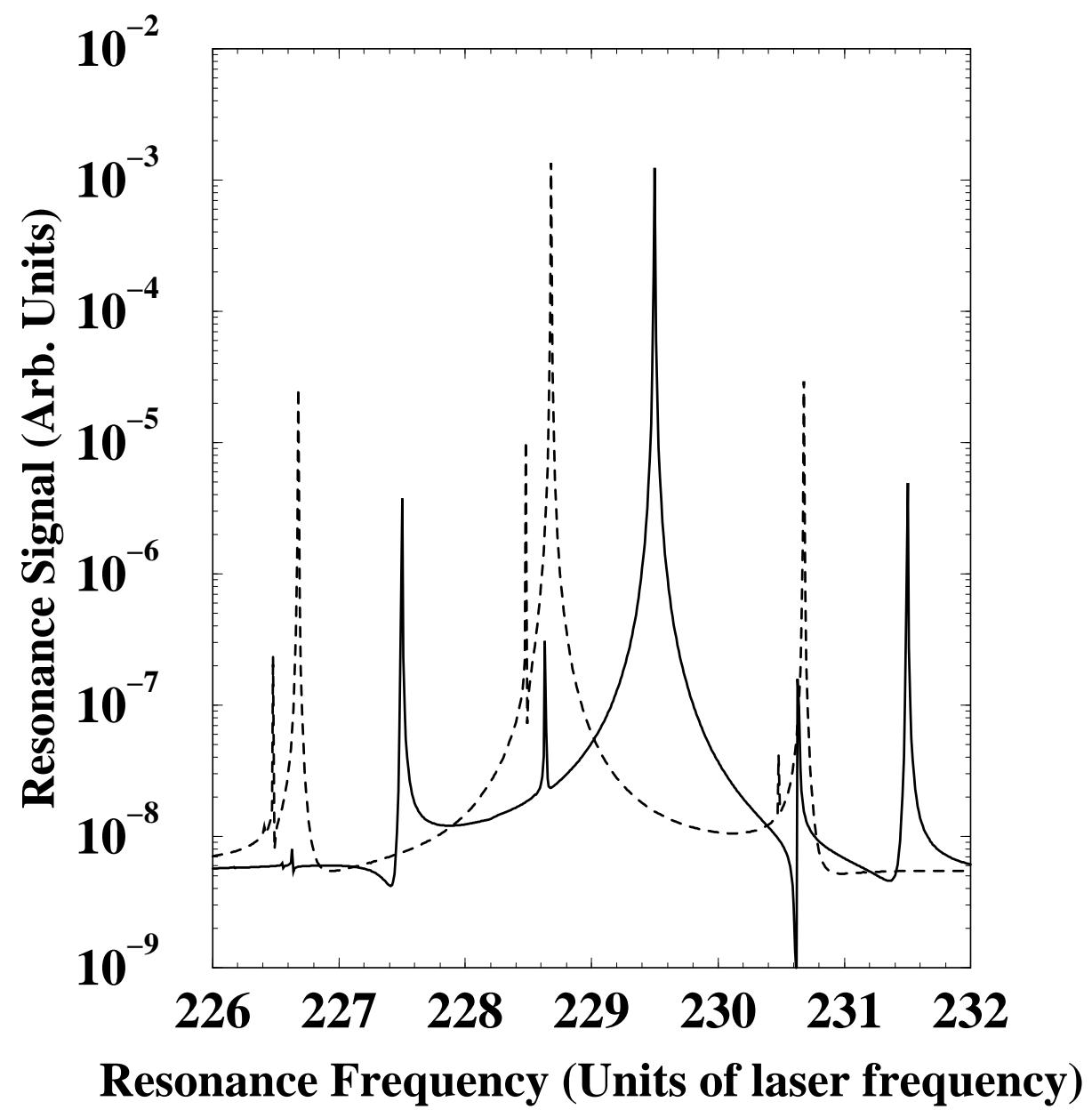

Fig. 8: S. X. Hu and C. H. Keitel, "Dynamics of ..." 

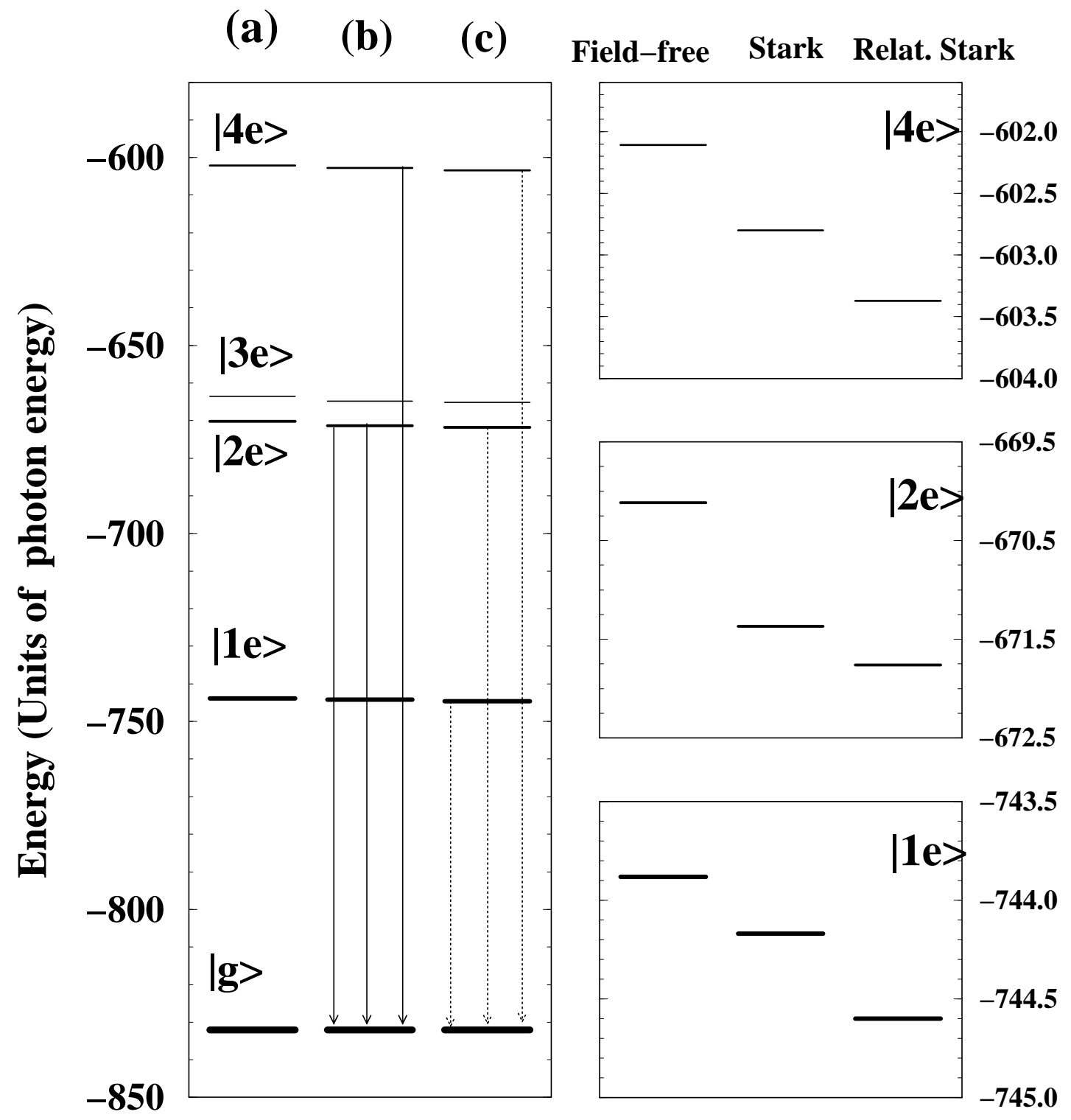

Fig. 9: S. X. Hu and C. H. Keitel, "Dynamics of ..." 

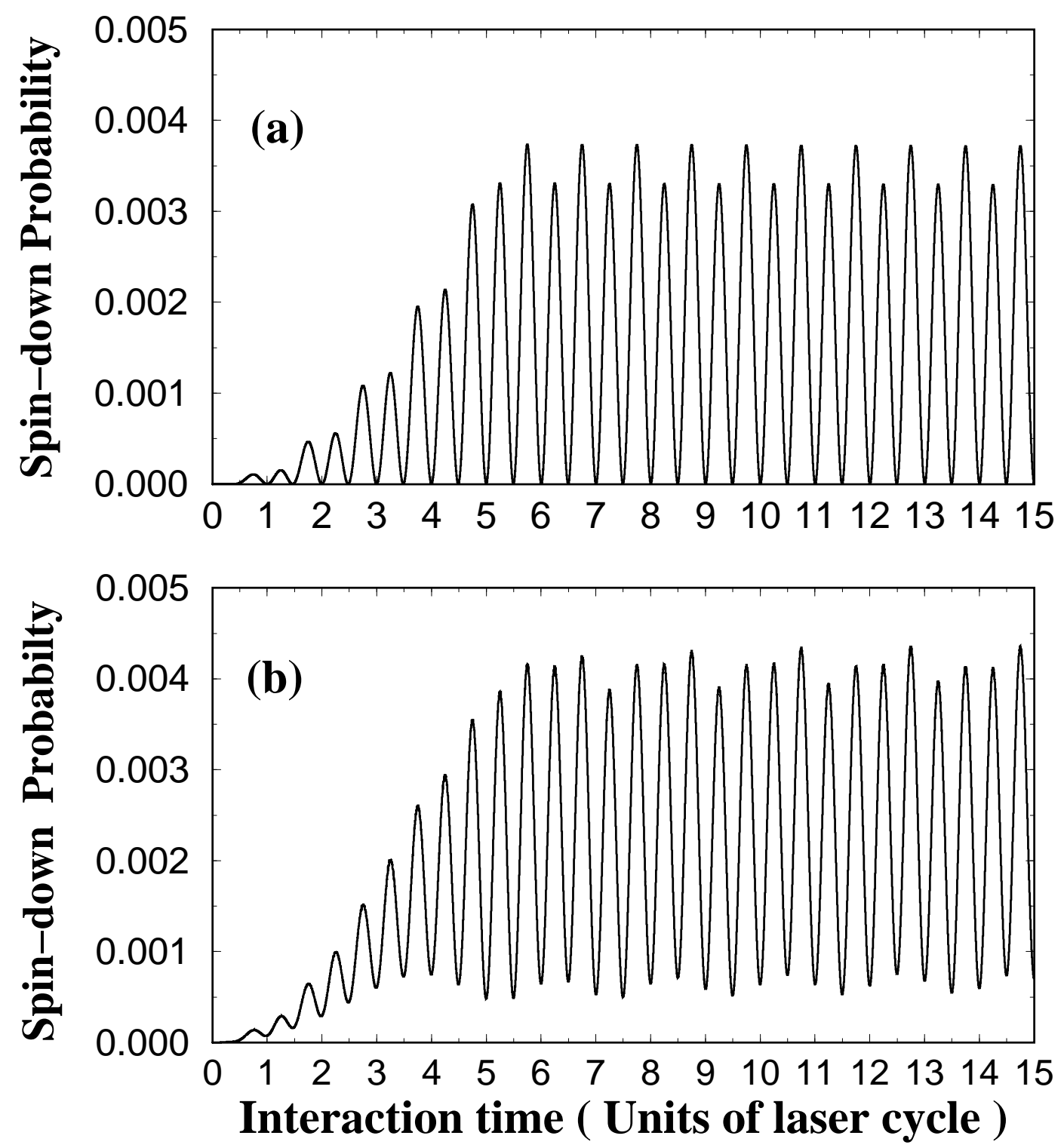

Fig. 10: S. X. Hu and C. H. Keitel, "Dynamics of ..." 
i) (a)
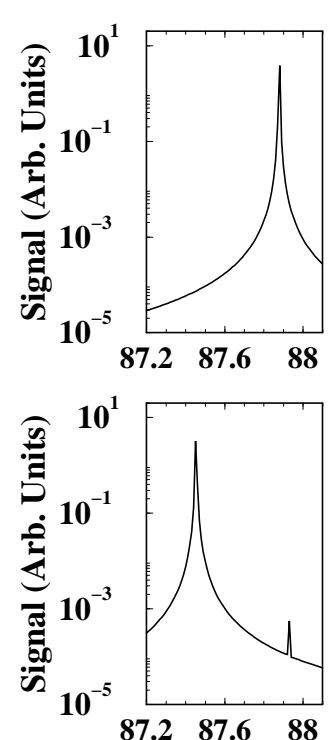

ii) Radiation frequency (Units of laser frequency)

(c)

$10^{-5}$

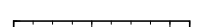

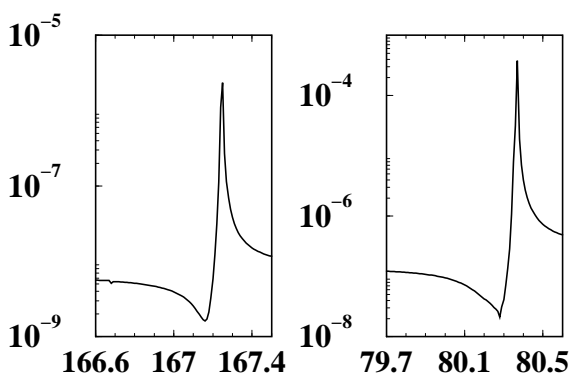
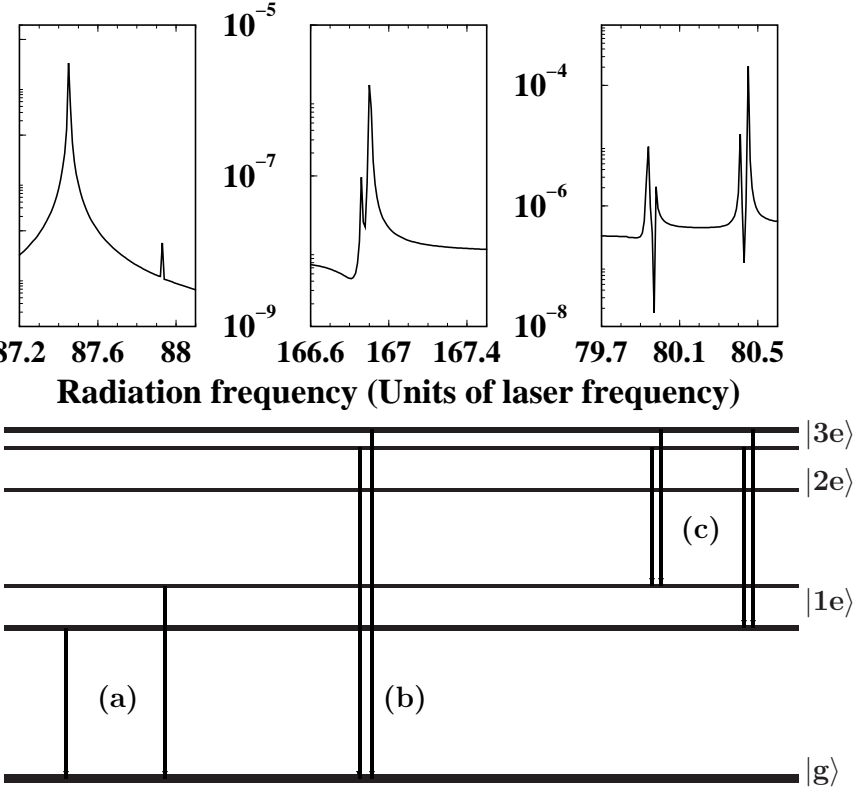

Fig. 11: S. X. Hu and C. H. Keitel, "Dynamics of ..." 


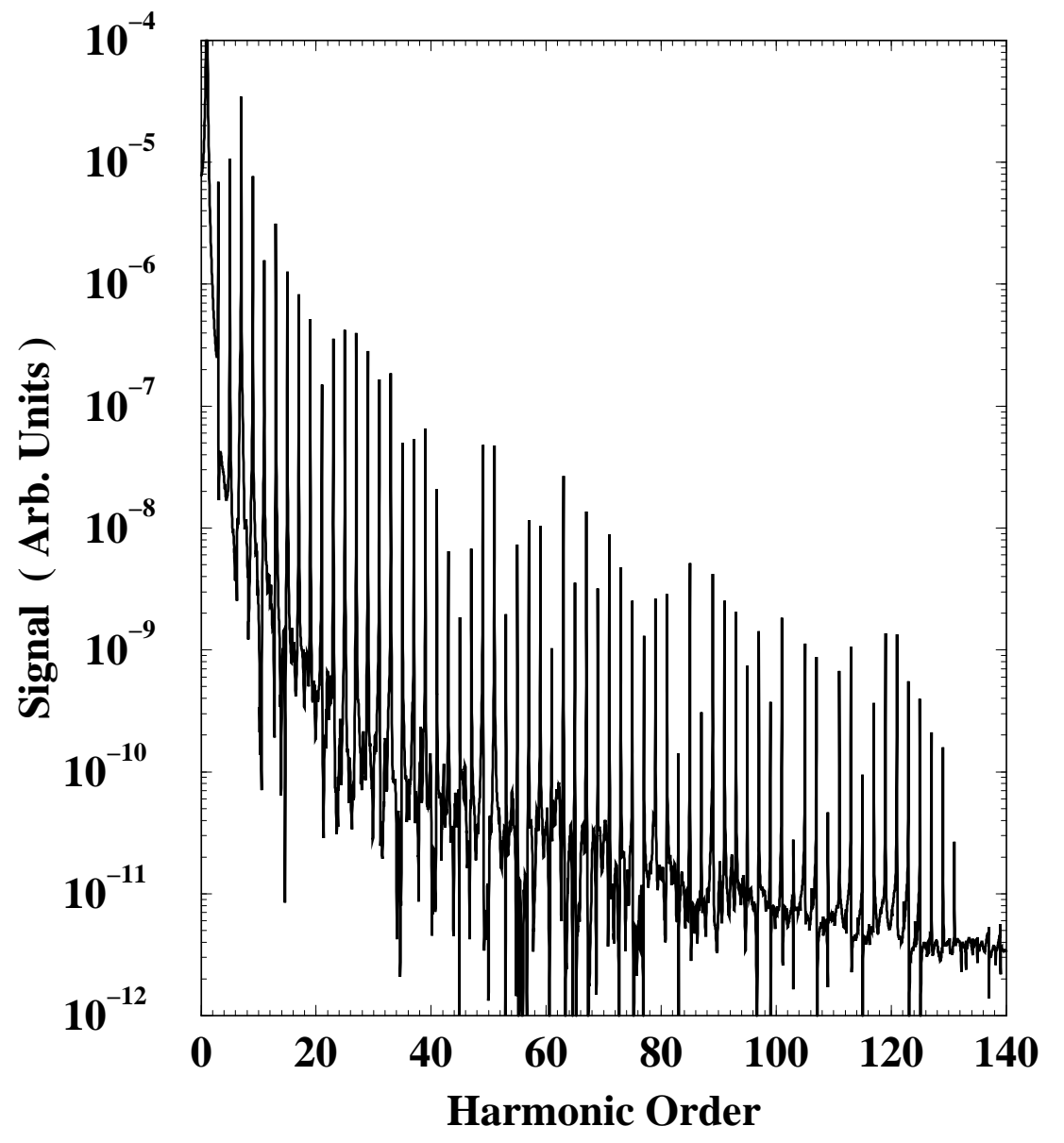

Fig. 12: S. X. Hu and C. H. Keitel, "Dynamics of ..." 


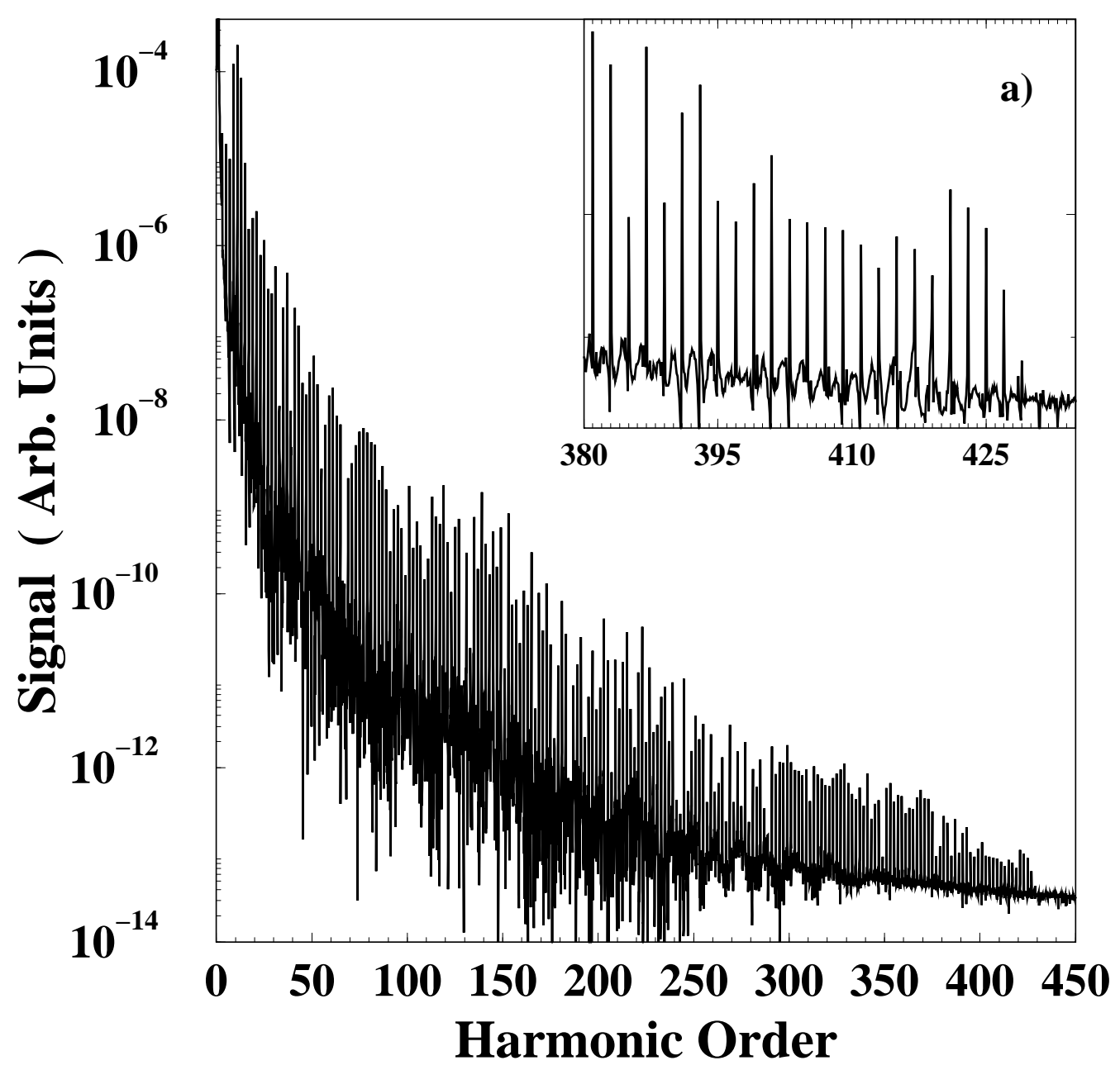

Fig. 13a: S. X. Hu and C. H. Keitel, "Dynamics of ..." 


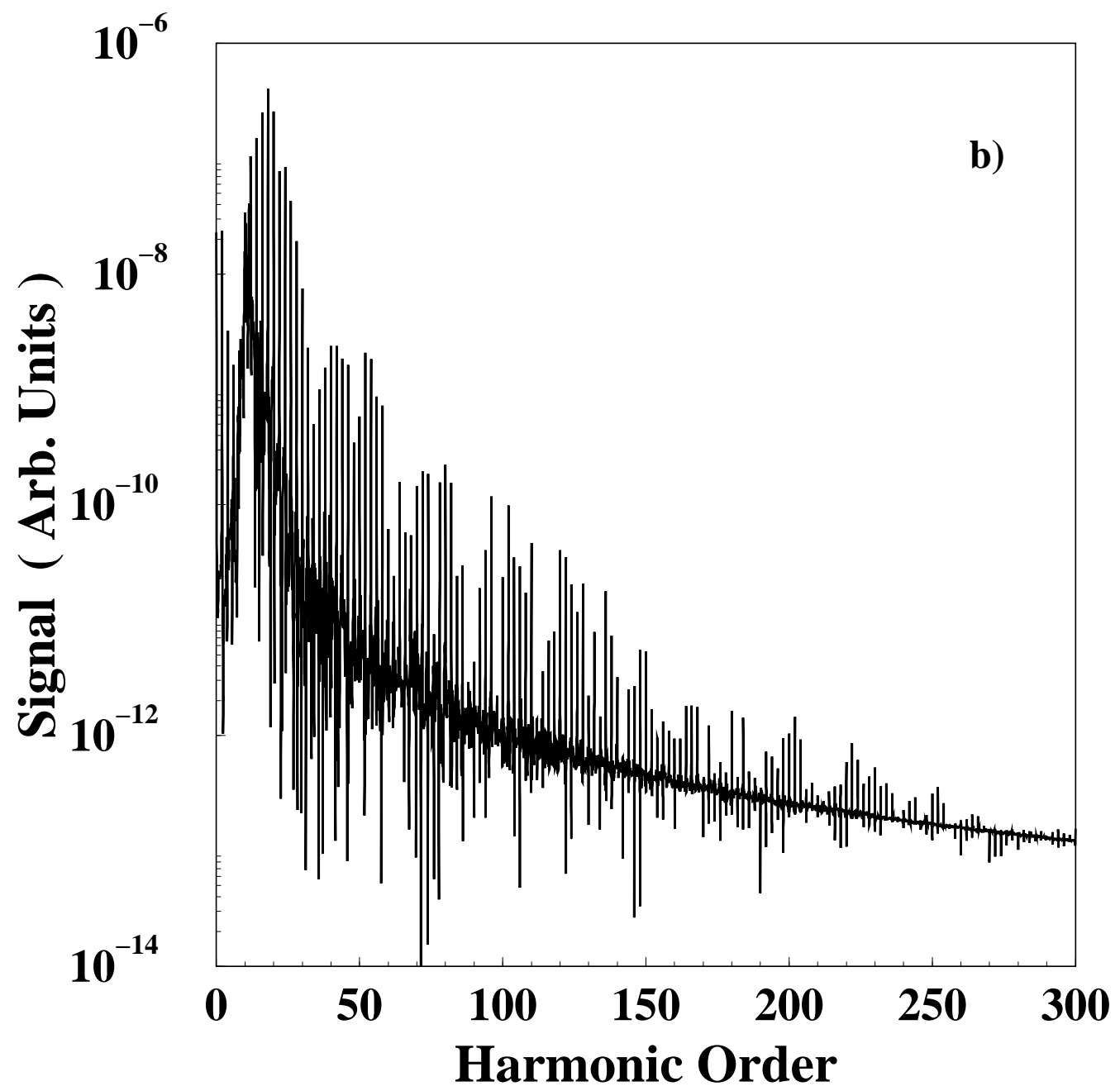

Fig. 13b: S. X. Hu and C. H. Keitel, "Dynamics of ..." 


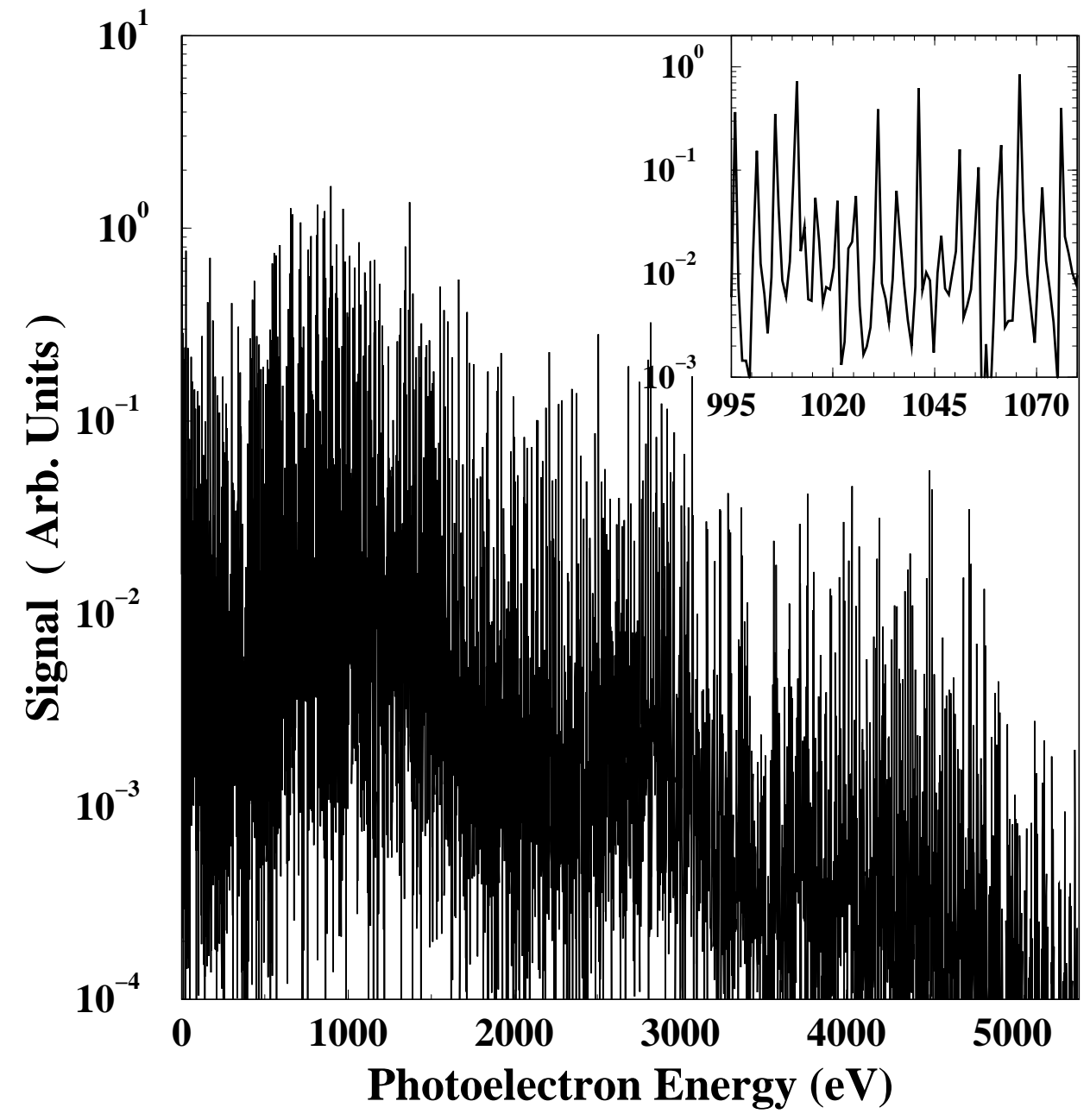

Fig. 14: S. X. Hu and C. H. Keitel, "Dynamics of ..." 


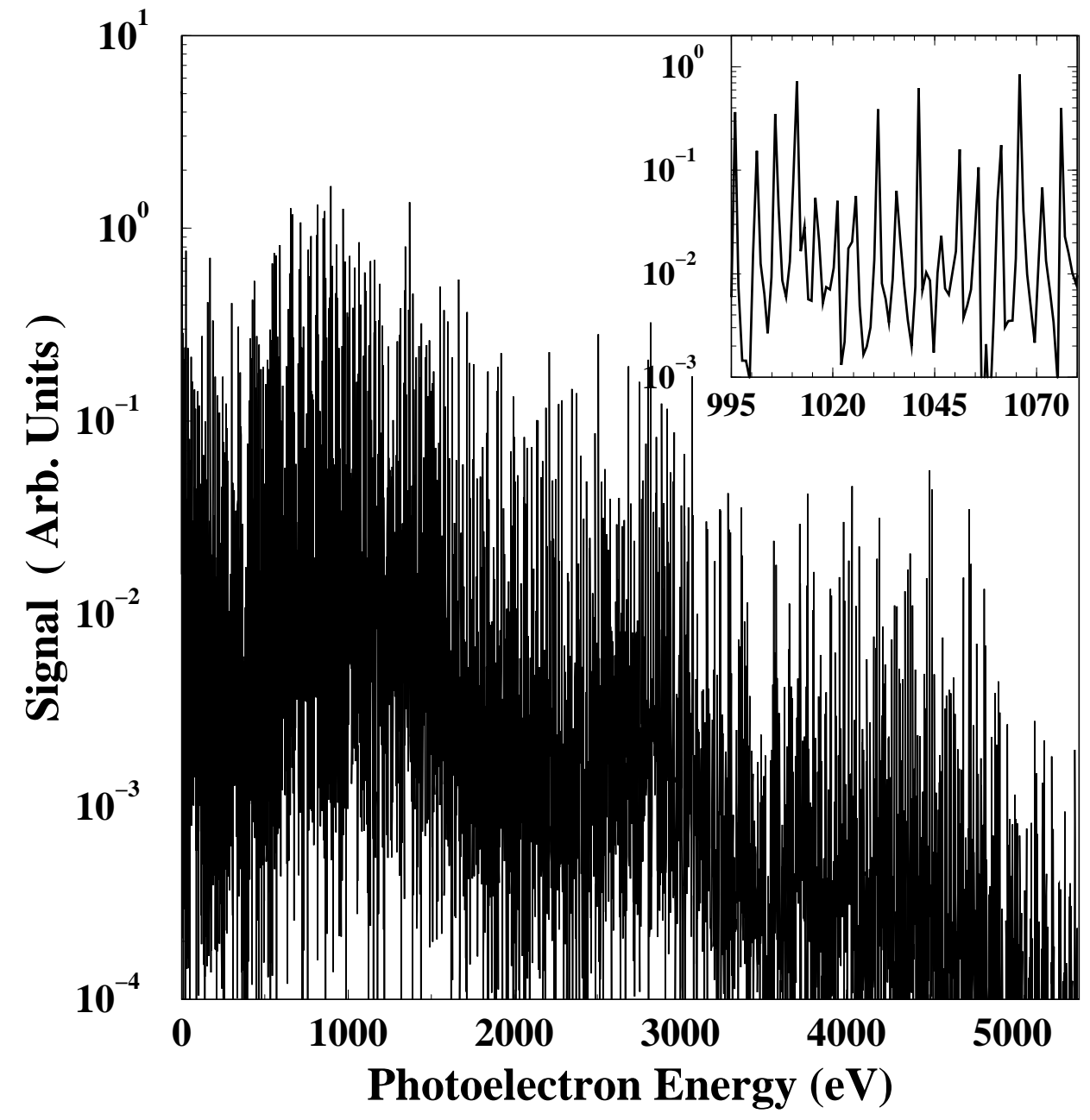

Fig. 15: S. X. Hu and C. H. Keitel, "Dynamics of ..." 